\title{
On the relationship between fuzzy autoepistemic logic and fuzzy modal logics of belief
}

\author{
Marjon Blondeel $^{\mathrm{d}, *, 1}$, Tommaso Flaminio ${ }^{\mathrm{a}, 2}$, Steven Schockaert ${ }^{\mathrm{b}}$, Lluís Godo ${ }^{\mathrm{c}, 3}$, \\ Martine De Cock ${ }^{\mathrm{d}, \mathrm{e}}$ \\ ${ }^{a}$ Università dell'Insubria, Department of Theorical and Applied Science, Via Mazzini 5, 21100 Varese, Italy \\ ${ }^{\mathrm{b}}$ Cardiff University, School of Computer Science and Informatics, 5 The Parade, Cardiff, CF24 3AA, UK \\ ${ }^{c}$ Artficial Intelligence Research Institute (IIIA) - CSIC, Campus UAB, 08193 Bellaterra, Spain \\ d Ghent University, Department of Applied Mathematics, Computer Science and Statistics, Krijgslaan 281 (S9), 9000 Gent, Belgium \\ ${ }^{\mathrm{e}}$ University of Washington Tacoma - Center for Web and Data Science, 1900 Commerce Street, Tacoma, WA 98402-3100, USA
}

Received 12 February 2014; received in revised form 30 September 2014; accepted 26 February 2015

\begin{abstract}
Autoepistemic logic is an important formalism for nonmonotonic reasoning originally intended to model an ideal rational agent reflecting upon his own beliefs. Fuzzy autoepistemic logic is a generalization of autoepistemic logic that allows to represent an agent's rational beliefs on gradable propositions. It has recently been shown that, in the same way as autoepistemic logic generalizes answer set programming, fuzzy autoepistemic logic generalizes fuzzy answer set programming as well. Besides being related to answer set programming, autoepistemic logic is also closely related to several modal logics. To investigate whether a similar relationship holds in a fuzzy logical setting, we firstly generalize the main modal logics for belief to the setting of finitely-valued Łukasiewicz logic with truth constants $€_{k}^{c}$, and secondly we relate them with fuzzy autoepistemic logics. Moreover, we show that the problem of satisfiability checking in these logics is NP-complete. Finally, we generalize Levesque's results on stable expansions, belief sets, and "only knowing" operators to our setting, and provide a complete axiomatization for a logic of "only knowing" in the $\mathrm{E}_{k}^{c}$ framework.
\end{abstract}

(C) 2015 Published by Elsevier B.V.

\footnotetext{
* Corresponding author.

E-mail addresses: marjon.blondeel@ugent.be (M. Blondeel), tommaso.flaminio@uninsubria.it (T. Flaminio), S.Schockaert@cs.cardiff.ac.uk (S. Schockaert), godo@iiia.csic.es (L. Godo), Martine.DeCock@UGent.be (M. De Cock).

1 Funded by a joint Research Foundation-Flanders (FWO) project (3G035612).

2 Partially funded by the Italian project FIRB 2010 (RBFR10DGUA-002).

3 Partially funded by the Spanish projects EdeTRI (TIN2012-39348-C02-01) and AT (CONSOLIDER CSD2007-0022, INGENIO 2010).
} 


\section{Introduction}

Since its introduction in the 1980s, autoepistemic logic [1-3] has been one of the main formalisms for nonmonotonic reasoning. It extends propositional logic by offering the ability to reason about an agent's (lack of) beliefs. In autoepistemic logic, beliefs are represented by sets of sentences in a propositional language augmented by a modal operator $\mathrm{B}$. If $\varphi$ is a formula, then $\mathrm{B} \varphi$, which has to be interpreted as " $\varphi$ is believed", is a formula as well. Note that in this language nested modal operators are allowed; it is possible to have beliefs about beliefs. Autoepistemic logic is nonmonotonic because conclusions made on the basis of incomplete information can be revised when new information becomes available. For instance, in autoepistemic logic we can express that "If I do not believe $p$, then $q$ is true." If $p$ cannot be inferred, $q$ can be derived, but when $p$ is added to the set of premises, $q$ can no longer be derived. Originally, autoepistemic logic was intended to model the beliefs of an ideal rational agent [1], by means of the so-called stable expansions. These are the deductively closed sets of beliefs that an ideal rational agent can have based on a particular set of premises $A$. Formally, given a set of autoepistemic formulas $A$, a set of autoepistemic formulas $E_{A}$ is a stable expansion of $A$ if

$$
E_{A}=\left\{\varphi \mid A \cup\left\{\mathrm{B} \psi \mid \psi \in E_{A}\right\} \cup\left\{\neg \mathrm{B} \psi \mid \psi \notin E_{A}\right\} \vdash \varphi\right\},
$$

where $\vdash$ denotes derivability in classical propositional logic, and each formula $\mathrm{B} \varphi$ is considered as a new propositional variable (e.g. $\mathrm{B}(a \wedge \mathrm{B} b)$ is a variable but $a \wedge \mathrm{B} b$ is the conjunction of the variables $a$ and $\mathrm{B} b)$. Using the definition of a stable expansion, it is easy to see that autoepistemic logic is nonmonotonic. Indeed, consider a set of premises $A=\{\neg \mathrm{B} p \rightarrow q\}$ and suppose there exists a stable expansion $E_{A}$. Since there is no reason to have $p \in E_{A}$, it follows that $\neg \mathrm{B} p \in E_{A}$ and thus $q \in E_{A}$. If $p$ is added to the premises: $A^{\prime}=\{\neg \mathrm{B} p \rightarrow q, p\}$, then $\mathrm{B} p \in E_{A^{\prime}}$ for a stable expansion $E_{A^{\prime}}$ and thus $q \notin E_{A^{\prime}}$.

In [4], a corresponding possible-world-like semantics is proposed. In particular, if we consider a set of propositional variables $V$, truth values are defined relatively to a pair $(I, S)$ where $S$ is a subset of classical interpretations in $\Omega=2^{V}$, representing the agent's beliefs (i.e. the agent's epistemic state), and $I \in \Omega$ is a particular interpretation intuitively corresponding to the "actual world". Note that, in contrast to the approach from [5], the approach from [4] does not require that $I \in S$, in which case $S$ models belief rather than knowledge. In fact, the pair $(I, S)$ can be regarded as a pointed S5 Kripke model (with accessibility relation $R=S \times S$ ) in case $I \in S$, or as a pointed KD45 Kripke model in case $I \notin S$. It is then shown that these semantics are in one-to-one correspondence with the stable expansions as originally defined in [1].

In [6], Levesque's logic of only knowing is introduced as an extension of autoepistemic logic to enable the modeling of expressions of the form " $\varphi$ is all that is believed", i.e. there are no other relevant beliefs, but $\varphi$. To this end, the language is expanded with a second modal operator O. For every formula $\varphi, \mathrm{O} \varphi$ reads " $\varphi$ is all that is believed" or "only $\varphi$ is believed". In [6] it is then shown that stable expansions correspond to a particular type of valid sentences in this logic. Finally, a sound and complete axiomatization based on classical K45 modal logic is provided by pointing out that $\mathrm{O} \varphi$ can be rewritten as $\mathrm{B} \varphi \wedge \mathrm{N} \neg \varphi$ where the modal operators $\mathrm{N}$ and $\mathrm{B}$ are both $\mathrm{K} 45$ operators. In particular, $\mathrm{B} \varphi$ corresponds to " $\varphi$ is believed" and $\mathrm{N} \neg \varphi$ to "at most $\neg \varphi$ is believed to be false". Hence $\mathrm{O} \varphi$ corresponds to "at least and at most $\varphi$ is believed", i.e. "exactly $\varphi$ is believed".

Logic programming had a significant impact on the development of some nonmonotonic logics and vice-versa (e.g. [7]). In particular, Gelfond and Lifschitz [8] showed that there is a one-to-one correspondence between the answer sets of an answer set program and the stable expansions of a corresponding autoepistemic theory. Hence, answer sets also correspond to valid sentences in logic of only knowing. Recently, a fuzzy generalization of autoepistemic logic has been defined in [9]. In the same paper, it has also been shown that the important relation between autoepistemic logic and answer set programming is preserved: the answer sets of a fuzzy answer set program (e.g. [10]) can be equivalently determined by computing the stable fuzzy expansions of a corresponding set of fuzzy autoepistemic formulas.

In this paper, in order to model the notion of belief on fuzzy propositions, we introduce generalizations of the main classical propositional modal logics of belief (K45, KD45, S5) based on finitely-valued Łukasiewicz logic with truth constants. Throughout this paper, we will denote the $k+1$ valued Łukasiewicz logic with truth constants as $\complement_{k}^{c}$. For technical reasons we only consider truth degrees belonging to a finite scale $S_{k}=\left\{0, \frac{1}{k}, \ldots, \frac{k-1}{k}, 1\right\}$. Indeed, this assumption ensures that two key properties are satisfied: $\complement_{k}^{c}$ is complete for deductions from an arbitrary set of premises, 


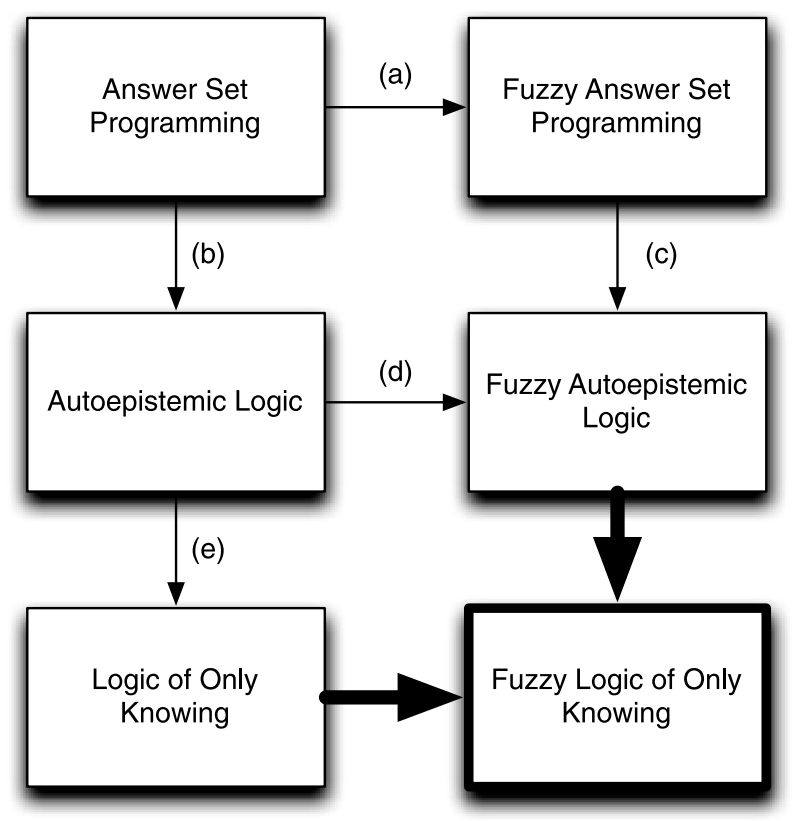

Fig. 1. (a): e.g. [10], (b): [8], (c)-(d): [9], (e): [6].

and the Baaz-Monteiro projection operator $\Delta$ is definable in $Ł_{k}^{c}$. Moreover, note that all operations that are definable in $(k+1)$-valued Gödel logic are definable in $(k+1)$-valued Łukasiewicz logic as well. Hence the choice for Łukasiewicz logic ensures some level of generality. Another possibility would be to consider BL [11], extended with the Baaz-Monteiro projection, but then the connectives lack a clear numerical interpretation.

Then, we show how fuzzy autoepistemic logic can also be characterized in terms of a possible worlds semantics intimately related to the Kripke models underlying the semantics of the $\mathfrak{七}_{k}^{c}$-variants of K45, KD45, and S5. We will show NP-completeness for two variants of the satisfiability problem. Finally, we also consider the extension of many-valued autoepistemic logic with an "only knowing" operator $\mathrm{O}$ and show that the relationship between stable expansions, belief sets and "only knowing" operators [6] naturally extends to our framework. As in the classical case we show that formulas of the form $\mathrm{O} \varphi$ can be rewritten as $\mathrm{B} \varphi \wedge \mathrm{N} \neg \varphi$ where $\mathrm{B}$ and $\mathrm{N}$ are many-valued K45 modalities. We provide a sound and complete axiomatization for this finitely-valued Łukasiewicz logic of only knowing and show that stable fuzzy expansions correspond to valid sentences in this logic. In particular this implies that the answer sets of a fuzzy answer set program correspond to valid sentences in this logic.

A summarizing diagram of embeddings and generalizations with references can be found in Fig. 1.

This paper is structured as follows. After this introduction, in Section 2, we recall some necessary preliminaries on the $(k+1)$-valued Łukasiewicz logic with truth constants $\mathfrak{七}_{k}^{c}$, and some results on the minimal modal logic over $€_{k}^{c}$. In Section 3, we define proper generalizations of the classical modal systems K45, KD45 and S5 and prove soundness and completeness with respect to appropriate Kripke-style semantics. In the same section, we also introduce a (possibly exponential) reduction for satisfiability from fuzzy to classical modal logics. In Section 4, we deal with the complexity of these logics and prove NP-completeness for two variants of the satisfiability problem. Then, in Section 5, we consider possible world semantics for the fuzzy autoepistemic logic of [9] and provide a characterization of stable fuzzy expansions both in terms of many-valued K45 belief sets, and in terms of proper generalizations of stable sets. In Section 6, we generalize the propositional fragment of Levesque's "only knowing" logic, we provide a sound and complete axiomatization, and we characterize stable fuzzy expansions in terms of the belief sets involving the "only knowing" operator $\mathrm{O}$. We conclude with related works and some final remarks. 


\section{Background}

\subsection{Finitely valued Łukasiewicz logic with truth constants}

Let $\mathcal{L}$ be the propositional language whose formulas are built from a countable set of propositional variables $V$, the binary connective $\rightarrow$ (implication), and truth constants $\bar{c}$ for each $c \in S_{k}=\left\{0, \frac{1}{k}, \ldots, \frac{k-1}{k}, 1\right\}$ (for some fixed $k \in \mathbb{N}$ ). Further connectives are defined as follows:

$$
\begin{aligned}
\neg \phi & =\phi \rightarrow \overline{0} & \phi \wedge \psi & =\phi \otimes(\phi \rightarrow \psi) \\
\phi \otimes \psi & =\neg(\phi \rightarrow \neg \psi) & \phi \oplus \psi & =\neg(\neg \phi \otimes \neg \psi) \\
\phi \vee \psi & =((\phi \rightarrow \psi) \rightarrow \psi) & \phi \leftrightarrow \psi & =(\phi \rightarrow \psi) \otimes(\psi \rightarrow \phi)
\end{aligned}
$$

with $\phi$ and $\psi$ arbitrary formulas. A propositional evaluation is a mapping $e: V \rightarrow S_{k}$ that extends to formulas in the following manner. If $\phi$ and $\psi$ are formulas and $c$ is an element in $S_{k}$, then

$$
e(\phi \rightarrow \psi)=e(\phi) \Rightarrow e(\psi) \text { and } e(\bar{c})=c,
$$

where $x \Rightarrow y=\min (1,1-x+y)$ for $x, y \in S_{k}$. Note that $(x \Rightarrow y)=1$ iff $x \leq y$. The set of all such evaluations will be denoted by $\Omega_{k}$. Notice that, in particular, for every formula $\phi$ and $\psi$ and for every $e \in \Omega_{k}$, we have

$$
\begin{aligned}
e(\neg \phi) & =1-e(\phi) & e(\phi \wedge \psi) & =\min (e(\phi), e(\psi)) \\
e(\phi \otimes \psi) & =\max (e(\phi)+e(\psi)-1,0) & e(\phi \oplus \psi) & =\min (1, e(\phi)+e(\psi)) \\
e(\phi \vee \psi) & =\max (e(\phi), e(\psi)) & e(\phi \leftrightarrow \psi) & =1-\mid(e(\phi)-e(\psi) \mid
\end{aligned}
$$

A formula $\phi$ is said to be satisfiable if there exists an $e \in \Omega_{k}$ such that $e(\phi)=1$. In such a case we say that $e$ is a model of $\phi$ and $e$ is a model of a set of formulas $T$ if $e$ is a model of every formula in $T$. A tautology is a formula $\phi$ such that $e(\phi)=1$ for each $e \in \Omega_{k}$. A formula $\phi$ is a semantic consequence of a set of formulas $\Gamma$, written as $\Gamma \models \phi$, if it holds that every model of $\Gamma$ is also a model of $\phi$.

This logic based on the language $\mathcal{L}$, which we will denote by $\mathfrak{七}_{k}^{c}$, has a sound and a strongly complete axiomatization, see e.g. [12] for details. In particular, the axioms of $\biguplus_{k}^{c}$ are

(Ł1) $\varphi \rightarrow(\psi \rightarrow \varphi)$,

(Ł2) $(\varphi \rightarrow \psi) \rightarrow((\psi \rightarrow \chi) \rightarrow(\varphi \rightarrow \chi))$,

(Ł3) $((\varphi \rightarrow \overline{0}) \rightarrow(\psi \rightarrow \overline{0})) \rightarrow(\psi \rightarrow \varphi)$,

(Ł4) $((\varphi \rightarrow \psi) \rightarrow \psi) \rightarrow((\psi \rightarrow \varphi) \rightarrow \varphi)$,

(Ł5) $(k+1) \varphi \leftrightarrow k \varphi$,

(Ł6) $\left(n \varphi^{n-1}\right)^{k+1} \leftrightarrow(k+1) \varphi^{n}$ for each natural number $n \in\{2, \ldots, k-1\}$ that does not divide $k$,

(Q1) $\left(\overline{c_{1}} \rightarrow \overline{c_{2}}\right) \leftrightarrow \overline{\min \left\{1,1-c_{1}+c_{2}\right\}}$,

and the only deduction rule is modus ponens (from $\phi$ and $\phi \rightarrow \psi$ infer $\psi$ ). Axioms (Ł1)-(Ł4) form an alternative axiomatization for Łukasiewicz logic, and in axioms (Ł5) and (Ł6), $n \varphi$ is an abbreviation for $\varphi \oplus \ldots \oplus \varphi$ (with $n$ repetitions of $\varphi)$ and $\varphi^{n}$ for $\varphi \otimes \ldots \otimes \varphi(n$ repetitions of $\varphi)$. From axiom (Q1) - the so called bookkeeping axiom we derive the canonical interpretation for truth constants.

Let $\vdash$ denote the deducibility relation in $\succeq_{k}^{c}$. Then, as usual, a formula $\psi$ can be derived from a set of formulas $T$, written $T \vdash \psi$, if there exists a finite sequence of formulas whose last member is $\psi$ and for which every element in the sequence is either (i) an instantation of an axiom in $\mathrm{七}_{k}^{c}$, (ii) an element in $T$, or (iii) a formula derived from previous elements in the sequence by modus ponens. The following result, which is considered to be folklore of the field, can be easily proved as a direct consequence of [13] and [14, Corollary 3.4].

Theorem 1 (Strong completeness). Let $T \cup\{\psi\}$ be an arbitrary set of formulas of $£_{k}^{c}$. Then $T \vdash \psi$ iff $T \models \psi$.

A formula $\psi$ that can be proven from the axioms of $\succeq_{k}^{c}$ and modus ponens is called a theorem; in this case we will write $\vdash \psi$. 
For each formula $\phi$ we will denote by $\Delta \phi$ the formula $\phi \otimes \ldots \otimes \phi$ (with $k$ repetitions of $\phi$ ). Since we only have $k+1$ truth values this formula is Boolean. Indeed, it is easy to check that for every $e \in \Omega_{k}$,

$$
e(\Delta \phi)= \begin{cases}1 & \text { if } e(\phi)=1 \\ 0 & \text { if } e(\phi)<1\end{cases}
$$

Note that $\Delta$ corresponds to the well-known Baaz-Monteiro projection operator which was introduced independently by Monteiro [15] and Baaz [16]. In semantical terms, a many-valued formula $\phi$ is transformed to a Boolean one $\Delta \phi$ which is false whenever $\phi$ is not fully true. In other words, if we consider a formula as a fuzzy set of evaluations $v_{\phi}(e)=e(\phi)$, then the $\Delta$ operator takes only the core of this fuzzy set.

\subsection{Minimal modal logic over $\varkappa_{k}^{c}$}

The minimal modal language $\mathcal{L}_{\mathrm{B}}$ we will consider in the paper is obtained by adding the modal operator B denoting "belief" to the language $\mathcal{L}$. Our approach is based on previous theoretical work [17] on fuzzy modal logics where the truth values are taken from a finite residuated lattice. In [17], among other logics, the authors introduce the minimal modal logic over $\mathfrak{七}_{k}^{c}$.

A semantics for $\mathcal{L}_{B}$ can be defined w.r.t. Kripke models of the form $M=(W, e, R)$ where $W$ is a countable set of possible worlds, $e: W \times V \rightarrow S_{k}$ is a mapping giving an evaluation of variables $e(w, \cdot): V \rightarrow S_{k}$ for each possible world $w \in W$, and $R \subseteq W \times W$ is a (two-valued) accessibility relation on possible worlds. Given a Kripke model $M=(W, e, R)$ and a world $w \in W$, the truth value of a formula $\phi$ in $\mathcal{L}_{\mathrm{B}}$ is inductively defined as follows:

- If $\phi$ is a propositional variable $p$, then $\|\phi\|_{M, w}=e(w, p)$

- If $\phi$ is a truth constant $\bar{c}$, then $\|\phi\|_{M, w}=c$

- If $\phi=\mathrm{B} \psi$, then $\|\phi\|_{M, w}=\inf _{w^{\prime}:\left(w, w^{\prime}\right) \in R}\|\psi\|_{M, w^{\prime}}$

- If $\phi=\psi \rightarrow \gamma$, then $\|\phi\|_{M, w}=\left(\|\psi\|_{M, w} \Rightarrow\|\gamma\|_{M, w}\right)$

The definition of $\|\mathrm{B} \psi\|_{M, w}$ intuitively expresses that the belief degree of $\psi$ in a world $w \in W$ coincides with the minimum truth degree that $\psi$ takes in every world which is accessible from $w$. A formula $\phi$ is said to be satisfiable if there exists a Kripke model $M=(W, e, R)$ and a $w \in W$ such that $\|\phi\|_{M, w}=1$. In such a case we say that $M$ is a model of $\phi$. A set of formulas $T$ is satisfied by a Kripke model $M$ if every formula in $T$ is satisfied by $M$. It is called a tautology if for each Kripke model $M=(W, e, R)$ and for each $w \in W$ we have $\|\phi\|_{M, w}=1$. A formula $\phi$ is a semantic consequence of a set of formulas $\Gamma$, written as $\Gamma \models_{\mathrm{B}} \phi$, if $\|\psi\|_{M, w}=1$ for all $\psi \in \Gamma$, for all Kripke models $M=(W, e, R)$ and for all $w \in W$ implies $\|\phi\|_{M, w}=1$ for all Kripke models $M=(W, e, R)$ and for all $w \in W$.

Now, let us consider the logic $\mathrm{BE}_{k}^{c}$ whose axioms are:

$\left(\mathrm{E}_{k}^{c}\right)$ axioms of $\mathrm{七}_{k}^{c}$,

(B2) $(\mathrm{B} \varphi \wedge \mathrm{B} \psi) \rightarrow \mathrm{B}(\varphi \wedge \psi)$,

(B3) $\mathrm{B}(\bar{c} \rightarrow \varphi) \leftrightarrow(\bar{c} \rightarrow \mathrm{B} \varphi)$, for each $c \in S_{k}$,

(B4) $(\mathrm{B} \varphi \oplus \mathrm{B} \varphi) \leftrightarrow \mathrm{B}(\varphi \oplus \varphi)$,

(K) $\mathrm{B}(\phi \rightarrow \psi) \rightarrow(\mathrm{B} \phi \rightarrow \mathrm{B} \psi)$,

and whose rules are modus ponens (from $\phi$ and $\phi \rightarrow \psi$ infer $\psi$ ) and necessitation for B (if $\phi$ is a theorem then $\mathrm{B} \phi$ is a theorem as well).

Axioms (B2) and (K) are typical axioms in normal modal logics. Axioms (B3) and (B4) basically reflect the semantics of the operator B (an infimum over worlds) on constants and on the Łukasiewicz strong disjunction. (B3) could be roughly read as stipulating that one believes that the truth value of $\phi$ is at least $c$ iff one believes at least to the degree $c$ that $\phi$ is true. In other words, this axiom tells us how graded belief has to be interpreted. (B4) can be understood as expressing that the operator $\mathrm{B}$ is commuting with any increasing unary connective of the logic. Note that if we define $\bullet \varphi:=\varphi \oplus \varphi$, then axiom (B4) could be simply expressed as B $\bullet \varphi \leftrightarrow \bullet \mathrm{B} \varphi$; and the same holds for $\bullet^{\prime} \varphi:=\varphi \otimes \varphi$. The axiom $\bar{c} \leftrightarrow \mathrm{B} \bar{c}$ is again a graded generalization of a standard KD45 axiom, which expresses that one believes tautologies $\mathrm{B} \top$ and does not believe falsehoods $\neg \mathrm{B} \perp$. Our generalization expresses that the truth constant $\bar{c}$ is believed to degree $c$. 
In [17], the authors show that this axiomatization is sound and complete w.r.t. the above class of Kripke models that will be denoted $\mathbb{M}$.

\section{Fuzzy modal logics of belief: extensions of $\mathrm{BL}_{k}^{c}$}

When defining logics of belief, it is common to require a number of properties of the modal operator B which do not follow from the axioms of $\mathrm{BŁ}_{k}^{c}$. For instance, it is common to presume that the agent has both positive and negative introspective capabilities. This is captured in the classical case by the well-known axioms (4) and (5). Belief consistency is captured by axiom (D). Finally, when dealing with knowledge instead of beliefs modal axiom (T) can be added. In this paper, we will consider extensions of $\mathrm{B}_{k}^{c}$ which are obtained by adding some or all of these classical axioms.

(D) $\neg \mathrm{B} \neg \overline{1}$

(4) $\mathrm{B} \phi \rightarrow \mathrm{BB} \phi$

(5) $\neg \mathrm{B} \phi \rightarrow \mathrm{B} \neg \mathrm{B} \phi$

(T) $\mathrm{B} \phi \rightarrow \phi$

As in the classical case [18,19], we consider the following extensions of $\mathrm{BE}_{k}^{c}$ :

- $K 45\left(\mathfrak{七}_{k}^{c}\right): \mathrm{BŁ}_{k}^{c}$ plus axioms (4) and (5),

- $K D 45\left(\mathfrak{E}_{k}^{c}\right): \mathrm{BŁ}_{k}^{c}$ plus axioms (D), (4) and (5),

- $S 5\left(\mathrm{E}_{k}^{c}\right): \mathrm{BE}_{k}^{c}$ plus axioms (T), (4) and (5).

We will denote by $\vdash_{L}$ the notion of proof for any of the logics $L \in\left\{K 45\left(\mathrm{E}_{k}^{c}\right), K D 45\left(\mathrm{七}_{k}^{c}\right), S 5\left(\mathrm{七}_{k}^{c}\right)\right\}$. The next task is to prove completeness of these logics ${ }^{4}$ with respect to corresponding classes of many-valued Kripke models.

As was done for (fuzzy) autoepistemic logic [1,9], we will sometimes consider a formula $\phi$ in $\mathcal{L}_{\mathrm{B}}$ as a $\mathrm{B}$-free formula $\phi^{*}$ by treating subformulas of the form $\mathrm{B} \psi$ as new propositional variables. For instance, $\mathrm{B}(a \wedge \mathrm{B} b)$ is then seen as a fresh variable $p_{\mathrm{B}(a \wedge \mathrm{B} b)}$ and has in principle no connection to $\mathrm{B} b$. Formally, consider the propositional language $\mathcal{L}^{*}$ built from variables $V^{*}=V \cup\left\{p_{\mathrm{B} \phi} \mid \phi \in \mathcal{L}_{B}\right\}$, truth constants in $S_{k}$ and the connective $\rightarrow$. We define the translation function $(\cdot)^{*}$ from $\mathcal{L}_{\mathrm{B}}$ to $\mathcal{L}^{*}$ in the following manner: $p^{*}=p, \bar{c}^{*}=\bar{c},(\phi \rightarrow \psi)^{*}=\phi^{*} \rightarrow \psi^{*}$ and $(\mathrm{B} \phi)^{*}=p_{\mathrm{B} \phi}$ (with $p_{\mathrm{B} \phi}$ a new variable). For a set of formulas $A$, we define $A^{*}=\left\{\psi^{*} \mid \psi \in A\right\}$. The set of all propositional evaluations $e: V^{*} \rightarrow S_{k}$ will be denoted by $\Omega_{k}^{*}$.

As a first step, we show a relation between proving a formula $\psi$ in one of the modal extensions of $\mathrm{BE}_{k}^{c}$ and proving the corresponding B-free formula $\psi^{*} \in \mathcal{L}^{*}$ from a suitable theory in the propositional logic $\mathfrak{Ł}_{k}^{c}$ (but over the set of variables $\left.V^{*}\right)$.

Lemma 1. Let $L$ be any of the logics $K 45\left(E_{k}^{c}\right), K D 45\left(E_{k}^{c}\right)$, S5( $\left(_{k}^{c}\right)$. Suppose $T \cup\{\psi\}$ is a set of formulas from $\mathcal{L}_{\mathrm{B}}$ and let $\Lambda_{L}=\left\{\phi^{*} \vdash \vdash_{L} \phi\right\}$. Then it holds that $T \vdash_{L} \psi$ iff $T^{*} \cup \Lambda_{L} \vdash \psi^{*}$.

Proof. See Appendix A.

The proof of Lemma 1 is based on the fact that a proof in $L$ can be translated into a proof in $\biguplus_{k}^{c}$ and vice versa. The set $\Lambda_{L}$ represents the set of $(\cdot)^{*}$-translations of modal theorems of $L$. As the following example illustrates this set is needed to convert a proof in $L$ to a proof in $Ł_{k}^{c}$.

Example 1. Consider $T=\{\mathrm{B} a\}$. Then $T \vdash_{L} \mathrm{~B}(b \rightarrow a)$ where $b$ is an arbitrary variable. Indeed, by axiom (Ł1) it follows that $\vdash_{L} a \rightarrow(b \rightarrow a)$ and thus by necessitation it follows that $\vdash_{L} \mathrm{~B}(a \rightarrow(b \rightarrow a))$. By axiom $(\mathrm{K})$ we have

$$
\vdash_{L} \mathrm{~B}(a \rightarrow(b \rightarrow a)) \rightarrow(\mathrm{B} a \rightarrow \mathrm{B}(b \rightarrow a)),
$$

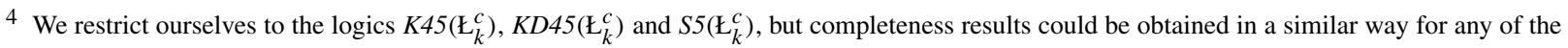
logics resulting from other combinations of the above axioms.
} 
and by modus ponens we can then infer $\vdash_{L} \mathrm{~B} a \rightarrow \mathrm{B}(b \rightarrow a)$. Another application of modus ponens using $\mathrm{B} a \in T$ then implies $T \vdash_{L} \mathrm{~B}(b \rightarrow a)$.

On the other hand, if we consider $T^{*}=\left\{p_{\mathrm{B}} a\right\}$, then we have $T^{*} \cup \Lambda_{L} \vdash p_{\mathrm{B}(b \rightarrow a)}$. Indeed, since $\vdash_{L} \mathrm{~B} a \rightarrow$ $\mathrm{B}(b \rightarrow a)$, it follows that

$$
p_{\mathrm{B}} a \rightarrow p_{\mathrm{B}(b \rightarrow a)}=(\mathrm{B} a \rightarrow \mathrm{B}(b \rightarrow a))^{*} \in \Lambda_{L}
$$

and by modus ponens and $p_{\mathrm{B}} a \in T^{*}$ we derive that $T^{*} \cup \Lambda_{L} \vdash p_{\mathrm{B}(b \rightarrow a)}$.

We now define the canonical Kripke model for a given fuzzy modal logic $L$. We will use this Kripke model to show completeness in Theorem 2. The following definition applies to any logic $L$ obtained by adding to the axioms of $\mathrm{BE}_{k}^{c}$ combinations of the axioms (D), (4), (5) and (T) and hence in particular for $L \in\left\{K 45\left(\mathrm{E}_{k}^{c}\right), K D 45\left(\mathrm{E}_{k}^{c}\right), S 5\left(\mathrm{E}_{k}^{c}\right)\right\}$. The $L$-canonical Kripke model is defined as the Kripke model $M_{c a n}^{L}=\left(W_{c a n}^{L}, e_{c a n}^{L}, R_{c a n}^{L}\right)$, where

- $W_{\text {can }}^{L}=\left\{w \in \Omega_{k}^{*} \mid \forall \phi \in \Lambda_{L}: w(\phi)=1\right\}$ with $\Lambda_{L}=\left\{\phi^{*} \mid \vdash_{L} \phi\right\}$,

- $R_{\text {can }}^{L}=\left\{\left(w_{1}, w_{2}\right) \in \Omega_{k}^{*} \times \Omega_{k}^{*} \mid \forall \phi \in \mathcal{L}_{\mathrm{B}}\right.$ : if $w_{1}\left((\mathrm{~B} \phi)^{*}\right)=1$, then $\left.w_{2}\left(\phi^{*}\right)=1\right\}$,

- $e_{\text {can }}^{L}(w, p)=w(p)$ for each variable $p$.

We now introduce some subclasses of the class $\mathbb{M}$ of Kripke models $(W, e, R)$ with two-valued accessibility relations, depending on which properties $R$ satisfies. Recall that a relation $R: X \times X \rightarrow\{0,1\}^{5}$ is

- Euclidean if $R(x, y)=R(x, z)=1$ implies $R(y, z)=1$.

- serial if for every $x \in X$ there exists $y \in X$ such that $R(x, y)=1$.

- transitive if $R(x, y)=R(y, z)=1$ implies $R(x, z)=1$.

- reflexive if $R(x, x)=1$ for all $x \in X$.

- symmetric if $R(x, y)=R(y, x)$ for all $x, y \in X$.

We then define the following subclasses of $\mathbb{M}$.

- $\mathbb{M}_{\mathrm{et}}$ : class of Kripke models $(W, e, R)$ with $R$ Euclidean and transitive.

- $\mathbb{M}_{\text {est }}$ : class of Kripke models $(W, e, R)$ with $R$ Euclidean, serial and transitive.

- $\mathbb{M}_{\text {rsyt }}$ : class of Kripke models $(W, e, R)$ with $R$ reflexive, symmetric and transitive.

In order to prove the main result of this section, i.e. Theorem 2, we first need first to prove the following preliminary lemma. This lemma shows the relation between evaluations of formulas in the $L$-canonical Kripke model, and evaluations of the corresponding $B$-free formulas in $\bigsqcup_{k}^{c}$.

Lemma 2. (Truth-lemma) Suppose $\phi$ is a formula in $\mathcal{L}_{\mathrm{B}}$ and $L \in\left\{K 45\left(\in_{k}^{c}\right), K D 45\left(€_{k}^{c}\right), S 5\left(\varkappa_{k}^{c}\right)\right\}$ with $M_{\text {can }}^{L}$ its canonical Kripke model. Then it holds that $v\left(\phi^{*}\right)=\|\phi\|_{M_{\text {can }}^{L}, v}$, for every $v \in W_{\text {can }}^{L}$.

Proof. By using the monotonicity for $\mathrm{B}$ and the distributivity of $\vee$ and $\wedge$, the claim follows by an easy adaptation from Lemma 4.20 in [17].

The following proposition collects basic properties of canonical Kripke models.

Proposition 1. Let $L \in\left\{K 45\left(E_{k}^{c}\right), K D 45\left(E_{k}^{c}\right), S 5\left(E_{k}^{c}\right)\right\}$, then the following conditions hold

1. If $L$ contains axiom $(T)$ then $R_{\text {can }}^{L}$ is reflexive.

2. If $L$ contains axiom (4) then $R_{\text {can }}^{L}$ is transitive.

\footnotetext{
5 Note that a relation $R \subseteq X \times X$ can be seen as a mapping $R: X \times X \rightarrow\{0,1\}$ where $R(x, y)=1$ iff $(x, y) \in R$. In this text will use both notations to denote two-valued relations.
} 
3. If $L$ contains axiom (5) then $R_{\text {can }}^{L}$ is Euclidean.

4. If $L$ contains axiom $(D)$ then $R_{\text {can }}^{L}$ is serial.

Proof. See Appendix A.

Using Proposition 1, we can now show the following theorem.

Theorem 2. $K 45\left({E_{k}^{c}}^{c}\right), K D 45\left(\biguplus_{k}^{c}\right)$ and $S 5\left(\biguplus_{k}^{c}\right)$ are sound and complete w.r.t. the classes $\mathbb{M}_{\mathrm{et}}, \mathbb{M}_{\mathrm{est}}$ and $\mathbb{M}_{\mathrm{rsyt}}$ respectively.

Proof. Soundness is straightforward. We can show the completeness by proving that if there is a formula $\phi$ such

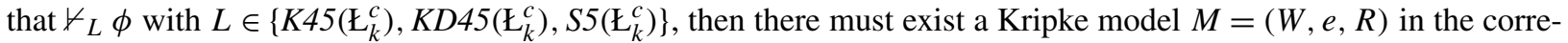
sponding subclass of Kripke models and a $w \in W$ such that $\|\phi\|_{M, w}<1$. We show that the $L$-canonical Kripke model meets this condition. The fact that each of these canonical Kripke models belongs to the correct subclass of $\mathbb{M}$ follows from Proposition 1 and from the fact that all reflexive and Euclidean relations are symmetric. By Lemma 1 it follows, independent of the choice of $L$, that $\Lambda_{L} \nvdash \phi^{*}$ and by the strong completeness of $\succeq_{k}^{c}$ it then follows that $\Lambda_{L} \not \models \phi^{*}$, i.e. there exists a $v \in W_{c a n}^{L}$ such that $v\left(\phi^{*}\right)<1$. By Lemma 2 we obtain that $\|\phi\|_{M_{c a n}, v}<1$.

As in the classical case (see e.g. [20]), the logics $K 45\left(\leftarrow_{k}^{c}\right), K D 45\left(\mathfrak{\leftarrow}_{k}^{c}\right)$ and $S 5\left(\mathfrak{\leftarrow}_{k}^{c}\right)$ admit simpler semantics while preserving soundness and completeness. Consider the following classes of Kripke models where $W \times E$ is the mapping $W \times W \rightarrow\{0,1\}$ such that $(W \times E)(w, v)=1$ if $(w, v) \in W \times E$, i.e. $v \in E$, and $(W \times E)(w, v)=0$ otherwise.

- $\mathbb{M}_{\mathrm{et}}^{s}$ : the subclass of Kripke models $M=(W, e, R)$ with $R=W \times E$ for some fixed $E \subseteq W$

- $\mathbb{M}_{\text {est }}^{s}$ : the subclass of Kripke models $M=(W, e, R)$ with $R=W \times E$ for some fixed and nonempty $E \subseteq W$

- $\mathbb{M}_{\text {rsyt }}^{s}$ : the subclass of Kripke models $M=(W, e, R)$ with $R=W \times W$

Notice that $\mathbb{M}_{\text {et }}^{s}, \mathbb{M}_{\text {est }}^{s}$ and $\mathbb{M}_{\text {rsyt }}^{s}$ are subclasses of resp. $\mathbb{M}_{\text {et }}, \mathbb{M}_{\text {est }}$ and $\mathbb{M}_{\text {rsyt }}$.

Proposition 2. $K 45\left(\leftarrow_{k}^{c}\right), K D 45\left(\leftarrow_{k}^{c}\right)$ and $S 5\left(€_{k}^{c}\right)$ are sound and complete w.r.t. the classes $\mathbb{M}_{\mathrm{et}}^{s}$, $\mathbb{M}_{\mathrm{est}}^{s}$ and $\mathbb{M}_{\mathrm{rsyt}}^{s}$ respectively.

Proof. See Appendix A.

Finally, let us show that one can reduce reasoning in any of our many-valued modal logics to reasoning in classical modal logics. We show it for the basic modal logic $\mathrm{BE}_{k}^{c}$.

For each formula $\phi \in \mathcal{L}_{B}$ and truth value $r \in S_{k}$ consider the formula $\phi_{r} \in \mathcal{L}_{B}$ defined as $\Delta(\phi \leftrightarrow \bar{r})$. This formula is a Boolean formula and for each Kripke model $M=(W, e, R)$ in $\mathbb{M}$ and each $w \in W$ we have $\left\|\phi_{r}\right\|_{M, w}=1$ iff $\|\phi\|_{M, w}=r$. Define $\mathcal{L}_{B}^{\prime} \subseteq \mathcal{L}_{\mathrm{B}}$ as the set of formulas constructed from the set of formulas $\left\{\Delta(p \leftrightarrow \bar{r}) \mid p \in V, r \in S_{k}\right\}$ :

- $\Delta(p \leftrightarrow \bar{r}) \in \mathcal{L}_{B}^{\prime}$ for every $p \in V$ and $r \in S_{k}$.

- $(\phi \rightarrow \psi) \in \mathcal{L}_{B}^{\prime}$ if $\phi, \psi \in \mathcal{L}_{B}^{\prime}$.

- $\mathrm{B} \phi \in \mathcal{L}_{B}^{\prime}$ if $\phi \in \mathcal{L}_{B}^{\prime}$.

In the following lemma we will show that for each formula $\Delta(\phi \leftrightarrow \bar{r})$, for which we will use the short notation $\phi_{r}$, there exists some $\left(\phi_{r}\right)^{\prime} \in \mathcal{L}_{B}^{\prime}$ such that the truth values remain the same.

Lemma 3. For any formula $\phi \in \mathcal{L}_{\mathrm{B}}$ and truth value $r \in S_{k}$, there exists a formula $\left(\phi_{r}\right)^{\prime} \in \mathcal{L}_{B}^{\prime}$ such that for each Kripke model $M=(W, e, R) \in \mathbb{M}$ and each world $w \in W$ it holds that

$$
\left\|\phi_{r}\right\|_{M, w}=\left\|\left(\phi_{r}\right)^{\prime}\right\|_{M, w} .
$$

Proof. See Appendix A. 
Example 2. Consider $S_{2}=\left\{0, \frac{1}{2}, 1\right\}, \phi=a \rightarrow b, \psi=\mathrm{B} a$ and $r=\frac{1}{2}$. Then e.g.

$$
\left(\phi_{r}\right)^{\prime}=\left(a_{\frac{1}{2}} \wedge b_{0}\right) \vee\left(a_{1} \wedge b_{\frac{1}{2}}\right)
$$

and

$$
\left(\psi_{r}\right)^{\prime}=\mathrm{B}\left(a_{\frac{1}{2}} \vee a_{1}\right) \wedge \neg \mathrm{B}\left(a_{1}\right) .
$$

Using Lemma 3 we then have the following reduction from $\mathrm{B}_{k}^{c}$ to classical modal logic.

Proposition 3. For each formula $\phi \in \mathcal{L}_{B}$ and $r \in S_{k}$ there exists some formula $\psi \in \mathcal{L}_{B}^{\prime}$, i.e. $\psi$ is a Boolean formula, such that

$$
\|\phi\|_{M, w}=r \text { iff }\|\psi\|_{M, w}=1
$$

for every Kripke model $M=(W, e, R) \in \mathbb{M}$ and each world $w \in W$.

\section{Complexity of satisfiability problems}

This section is concerned with the complexity of two satisfiability problems for $K D 45\left(\in_{k}^{c}\right)$. The results we will obtain also hold for $K 45\left(\mathrm{E}_{k}^{c}\right)$ and $S 5\left(\mathrm{E}_{k}^{c}\right)$ because the proofs we will show can easily be adapted to these cases.

Let us define the following variants of the classical SAT-problem:

- 1-SAT: Given a formula $\phi \in \mathcal{L}_{\mathrm{B}}$, does there exist an $M=(W, e, R) \in \mathbb{M}_{\text {est }}^{s}$ and a $w \in W$ such that $\|\phi\|_{M, w}=1$ ?

- pos-SAT: Given a formula $\phi \in \mathcal{L}_{\mathrm{B}}$, does there exist an $M=(W, e, R) \in \mathbb{M}_{\text {est }}^{s}$ and a $w \in W$ such that $\|\phi\|_{M, w}>0$ ?

We will show that these problems are NP-complete which is the same complexity class as the corresponding decision problem for classical KD45 (see [20] for more details on the complexity of classical modal logics).

Note that 1-SAT and pos-SAT can be polynomially reduced to each other. Indeed, a formula $\phi$ is "pos-SAT" if $\neg \Delta(\neg \phi)$ is "1-SAT" and a formula $\phi$ is "1-SAT" if $\Delta \phi$ is "pos-SAT". Hence it is sufficient to show the NPcompleteness of 1-SAT.

For any formula $\phi \in \mathcal{L}_{\mathrm{B}}$, we denote by \# $\phi$ its length:

- \# $\bar{c}=1$ for each $c \in S_{k}$ and $\# p=1$ for every $p \in V$.

- \#( $\phi \rightarrow \psi)=1+\# \phi+\# \psi$ and similar for the other connectives.

$-\#(\mathrm{~B} \phi)=1+\# \phi$.

For a formula $\phi \in \mathcal{L}_{\mathrm{B}}$, we denote by $d(\phi)$ its depth which is defined as usual by counting the nested occurrences of the modality B.

Example 3. Consider the formula $\phi=\mathrm{B} a \oplus \neg b$ with $a$ and $b$ variables. Then

$$
\#(\mathrm{~B} a \oplus \neg b)=1+\#(\mathrm{~B} a)+\#(\neg b)=1+1+\#(a)+1+\#(b)=5 .
$$

For $\varphi$ and $\psi$ propositional (i.e. B-free) formulas, we have: $d(\varphi)=d(\psi)=0, d(\mathrm{~B}(\varphi))=d(\mathrm{~B}(\varphi \oplus \psi))=d(\varphi \oplus$ $\mathrm{B}(\psi))=1, d(B(\varphi \oplus B(\psi)))=2$ and so forth.

Lemma 4. Let $\phi$ be a formula in $\mathcal{L}_{\mathrm{B}}$. Then for every model $M=(W, e, R) \in \mathbb{M}_{\text {est }}^{s}$, and for every $w \in W$, there exists a finite model $M^{\prime}=\left(W^{\prime}, e^{\prime}, R^{\prime}\right) \in \mathbb{M}_{\mathrm{est}}^{s}$ and a world $w^{\prime} \in W^{\prime}$ such that $\left|W^{\prime}\right| \leq \# \phi$ and $\|\phi\|_{M, w}=\|\phi\|_{M^{\prime}, w^{\prime}}$.

Proof. Consider a Kripke model $M=(W, e, R)$ with $R=W \times E(\emptyset \neq E \subseteq W)$ and $w \in W$. The aim is to find a finite set $W^{\prime}$, a non-empty subset $E^{\prime} \subseteq W^{\prime}$, a mapping $e^{\prime}: W^{\prime} \times V \rightarrow S_{k}$ and some $w^{\prime} \in W^{\prime}$ for which the claim holds.

Trivially, if $\phi$ is B-free, take $W^{\prime}=E^{\prime}=\{w\}, R^{\prime}=W^{\prime} \times E^{\prime}, w^{\prime}=w$, and take $e^{\prime}$ be defined by restricting $e$ : $W \times V \rightarrow S_{k}$ to $W^{\prime} \times V$. 
In general, if $d(\phi) \geq 1$ we proceed as follows. Let $\mathrm{B} \psi_{1}^{1}, \ldots, \mathrm{B} \psi_{i_{1}}^{1}$ be the subformulas of $\phi$ of depth 1 , whence each $\psi_{j}^{1}$ is B-free. Since the set of truth-values $S_{k}$ is finite, for each $\psi_{j}^{1}$ there exists a world $w_{j}^{1}$ such that

$$
\left\|\mathrm{B} \psi_{j}^{1}\right\|_{M, w}=\left\|\psi_{j}^{1}\right\|_{M, w_{j}^{1}}=w_{j}^{1}\left(\psi_{j}^{1}\right) .
$$

Letting $r_{1 j}=\left\|\mathrm{B} \psi_{j}^{1}\right\|_{M, w}$, replace each subformula $\mathrm{B} \psi_{j}^{1}$ by the corresponding truth constant $\overline{r_{1 j}}$ and, if $d=d(\phi)>1$, repeat the process for all levels $2, \ldots, d$. Let $E^{\prime}=\left\{w_{j}^{l} \mid 1 \leq l \leq d, 1 \leq j \leq i_{l}\right\}, W^{\prime}=\{w\} \cup E^{\prime}, w^{\prime}=w$ and let $e^{\prime}$ be defined by restricting $e: W \times V \rightarrow S_{k}$ to $W^{\prime} \times V$. Then, by construction, $\|\phi\|_{M, w}=\|\phi\|_{M^{\prime}, w^{\prime}}$. Moreover, $\left|W^{\prime}\right|=1+\sum_{l=1}^{d} i_{l} \leq \# \phi$.

Observe that the proof of Lemma 4 uses a procedure which, given a formula $\phi \in \mathcal{L}_{B}$, a Kripke model $M=$ $(W, e, R)$ and a $w \in W$, outputs a B-free formula $\phi^{M}$ such that $M \models \phi$ if and only if $\phi^{M}$ is 1-satisfiable in $\mathfrak{七}_{k}^{c}$. In particular, $\phi^{M}$ is obtained by substituting each of the subformulas of the form $B \psi$ in $\phi$ by the truth-constant $\bar{r}$ with $r=\|B \psi\|_{M, w}$.

Theorem 3. The problems 1-SAT and pos-SAT for KD45( ${\leftarrow_{k}^{c}}^{c}$ are NP-complete.

Proof. Since each formula of Łukasiewicz logic is in particular a formula of $\mathcal{L}_{B}$, and since 1-SAT (as well as pos-SAT) for $\mathrm{E}_{k}^{c}$ is NP-complete, the NP-hardness of our problem follows. In order to prove NP-membership, recall that from Lemma 4 a formula $\phi$ is 1-SAT in a model $M$ iff $\phi$ is 1-SAT in a finite model $M^{\prime}$ whose cardinality is polynomial in the length of $\phi$. Let us guess the model $M^{\prime}=\left(W^{\prime}, e^{\prime}, R^{\prime}\right)$ and let us also guess $w^{\prime} \in W^{\prime}$. Since $\left|W^{\prime}\right| \leq \# \phi$, these guesses are polynomial in $\# \phi$. Let $\phi^{M^{\prime}}$ be the formula of $\succeq_{k}^{c}$ obtained from $M^{\prime}, w^{\prime}$ and $\phi$ applying the procedure described above. Notice that $\# \phi^{M^{\prime}}$ is polynomial in $\# \phi$. Moreover, since $\left|W^{\prime}\right| \leq \# \phi$ the formula $\phi^{M^{\prime}}$ is obtained in a number of steps which is polynomial in \# $\phi$. From [21] it follows that checking if $\phi^{M^{\prime}}$ is 1-SAT (pos-SAT) in $\mathrm{七}_{k}^{c}$ is in $\mathrm{NP}$, and hence the NP-membership follows.

Remark 1. For each $r \in S_{k}$, let us define the decision problem $r$-SAT in the following way:

"Given a formula $\phi$, does there exist an $M=(W, e, R) \in \mathbb{M}_{\text {est }}^{s}$ and a $w \in W$ such that $\|\phi\|_{M, w}=r ?$ ?"

It turns out that, for every $r \in S_{k}, r$-SAT is NP-complete as well. Indeed, this problem is equivalent to 1-SAT for the formula $\Delta(\phi \leftrightarrow \bar{r})$ which is polynomial in $\phi$. Hence NP-membership follows. NP-hardness follows from the fact that for each $r>0$, 1-SAT can be reduced to $r$-SAT since a formula $\|\phi\|_{M, w}=1$ iff $\|\phi \otimes \bar{r}\|_{M, w}=r$. For $r=0$, we can reduce 1-SAT as follows: $\|\phi\|_{M, w}=1$ iff $\|\neg \phi\|_{M, w}=0$.

Note that in [22], it was shown that 1-SAT and pos-SAT for the minimal modal logic over $\mathfrak{七}_{k}$ with $S_{k}$-valued accessibility relations are PSPACE-complete.

\section{Relating fuzzy modal logic and fuzzy autoepistemic logic}

The aim of Moore's autoepistemic logic [1] is to characterize the set of beliefs of a rational agent with introspection capabilities by means of a set of simple properties it must fulfill. The language of fuzzy autoepistemic logic is the same as $\mathcal{L}_{\mathrm{B}}$, the modal logic with the belief operator $\mathrm{B}$, so $\mathrm{B} \varphi$ is still read as "the agent believes $\varphi$ ". The basic construct is concerned with the notion of a stable expansion $E$ of a set of initial beliefs $A$, briefly introduced in Section 1 . It can be seen as the closed set of beliefs of an ideal rational agent reflecting on his own beliefs. In this section we first consider a many-valued generalization of the notion of stable expansions from [9]. Then we introduce a possible world semantics for the resulting many-valued stable expansions and we show their relation to the fuzzy modal logics studied in Section 3. Finally we establish a link to a graded generalization of Stalnaker's notion of stable belief sets.

\subsection{Stable fuzzy expansions}

The following semantic definition of stable fuzzy expansions has been introduced in [9]. 
Definition 1. A stable fuzzy expansion of a set of $\mathcal{L}_{\mathrm{B}}$-formulas $A$ is a fuzzy set $E_{A}: \mathcal{L}_{\mathrm{B}} \rightarrow S_{k}$ that satisfies the following fixpoint condition:

$$
E_{A}(\phi)=\min \left\{v\left(\phi^{*}\right) \mid v \in \Omega_{k}^{*}, v \text { model of } A^{*} \cup\left\{(\mathrm{B} \psi)^{*} \leftrightarrow \overline{E_{A}(\psi)} \mid \psi \in \mathcal{L}_{\mathrm{B}}\right\}\right\} .
$$

Recall that $\overline{E_{A}(\psi)}$ denotes the truth constant corresponding to the membership value of $\psi$ in $E_{A}$ and that, for a formula $\phi \in \mathcal{L}_{B}, \phi^{*}$ is the corresponding B-free formula, see Section 3. Using the strong completeness of $\mathfrak{七}_{k}^{c}$, in particular the fact that for any set of $€_{k}^{c}$ formulas $T \cup\{\phi\}$ one has

$$
\min \{v(\phi) \mid v(\psi)=1 \text { for all } \psi \in T\}=\max \left\{r \in S_{k} \mid T \vdash \bar{r} \rightarrow \phi\right\},
$$

and thus one can rewrite the above semantic definition of stable fuzzy expansion of $A$ as a fuzzy set of formulas $E_{A}$ satisfying the following syntactic fixpoint condition:

$$
E_{A}(\phi)=\max \left\{r \in S_{k} \mid A^{*} \cup\left\{(\mathrm{B} \psi)^{*} \leftrightarrow \overline{E_{A}(\psi)} \mid \psi \in \mathcal{L}_{\mathrm{B}}\right\} \vdash \bar{r} \rightarrow \phi^{*}\right\} .
$$

Still, this condition can be equivalently rewritten as the following two joint conditions:

$$
\begin{gathered}
\underline{E_{A}}=\left\{\phi \in \mathcal{L}_{\mathrm{B}} \mid A^{*} \cup\left\{(\mathrm{B} \psi)^{*} \leftrightarrow \overline{E_{A}(\psi)} \mid \psi \in \mathcal{L}_{\mathrm{B}}\right\} \vdash \phi^{*}\right\}, \quad \text { and } \\
E_{A}(\phi)=\max \left\{r \in S_{k} \mid \bar{r} \rightarrow \phi \in \underline{E_{A}}\right\} .
\end{gathered}
$$

Notice that $\phi \in \underline{E_{A}}$ if and only if $E_{A}(\phi)=1$. In other words, $\underline{E_{A}}$ is the core of the fuzzy set $E_{A}$.

\subsection{Possible world semantics for stable fuzzy expansions}

Generalizing Moore's result [4], it is shown in [9] that stable fuzzy expansions can also be characterized by a fuzzy Kripke-style possible world semantics. In particular, a fuzzy autoepistemic structure is a pair $(w, S)$ with $w \in \Omega_{k}$ and $S \subseteq \Omega_{k}$. Informally, $w$ represents the actual world which is used to evaluate B-free formulas and $S$ represents all worlds considered possible (i.e. epistemic states) and is used to evaluate formulas of the form $\mathrm{B} \psi$. The class of such structures will be denoted by $\mathbb{M}^{\text {ae }}$. The degree of truth of an $\mathcal{L}_{\mathrm{B}}$-formula $\phi$ relative to a fuzzy autoepistemic structure $(w, S)$ is inductively defined as follows:

- If $\phi$ is a propositional formula from $\mathcal{L}$, then $\|\phi\|_{(w, S)}=w(\phi)$.

- If $\phi$ is a truth constant $\bar{c}$, then $\|\phi\|_{(w, S)}=c$.

- If $\phi=\mathrm{B} \psi$, then $\|\mathrm{B} \psi\|_{(w, S)}=\inf \left\{\|\psi\|_{(v, S)} \mid v \in S\right\}$.

- If $\phi=\psi \rightarrow \gamma,\|\phi\|_{(w, S)}=\left(\|\psi\|_{(w, S)} \Rightarrow\|\gamma\|_{(w, S)}\right)$.

Intuitively, one can think of $S$ as a set of possible worlds. Hence, the truth degree of $\mathrm{B} \varphi$ in $S$ is equal to the minimal truth degree to which $\varphi$ is considered to be true in all possible worlds. In other words, we can interpret the truth value of $\mathrm{B} \varphi$ as the minimal truth value for $\varphi$ which is supported by the agent's epistemic state.

Since the truth evaluation of formulas of the form $\mathrm{B} \varphi$ in a structure $(w, S)$ does not depend on the actual world $w$, we will also write $\|\mathrm{B} \varphi\|_{S}$ to denote $\|\mathrm{B} \varphi\|_{(w, S)}$. Note that, if $S=\emptyset$, then $\|\mathrm{B} \varphi\|_{S}=1$. Also note that, conversely, the interpretation $w$ in $(w, S)$ is needed to evaluate non-modal formulas.

We consider the following subclasses of fuzzy autoepistemic structures of $\mathbb{M}^{\text {ae }}$ :

- the class $\mathbb{M}_{e}^{\mathrm{ae}}$, where only pairs $(w, S)$ with $S$ non-empty are considered,

- the class $\mathbb{M}_{i n}^{a \mathrm{ae}} \subseteq \mathbb{M}_{e}^{\mathrm{aee}}$, where only pairs $(w, S)$ with $w \in S$ are considered.

It can be shown that $K 45\left(\mathrm{E}_{k}^{c}\right), K D 45\left(\mathrm{七}_{k}^{c}\right)$ and $S 5\left(\mathrm{E}_{k}^{c}\right)$ are still sound and complete with respect to the classes $\mathbb{M}^{\text {ae }}$, $\mathbb{M}_{e}^{\mathrm{ae}}$ and $\mathbb{M}_{\text {in }}^{\mathrm{ae}}$ respectively.

Theorem 4. $K 45\left(\leftarrow_{k}^{c}\right), K D 45\left({E_{k}^{c}}^{c}\right)$ and $S 5\left(\left(_{k}^{c}\right)\right.$ are sound and complete w.r.t. to $\mathbb{M}^{\mathrm{ae}}, \mathbb{M}_{e}^{\mathrm{ae}}$ and $\mathbb{M}_{i n}^{\mathrm{ae}}$, respectively. 
Table 1

List of languages, logics and semantics used in this paper.

\begin{tabular}{|c|c|c|c|}
\hline & Syntax & Logic & Semantics \\
\hline $\begin{array}{l}\text { Finitely valued Łukasiewicz } \\
\text { logic with truth constants over } \\
\text { variables } V\end{array}$ & $\mathcal{L}$ & $\mathfrak{E}_{k}^{c}$ & $\Omega_{k}$ \\
\hline $\begin{array}{l}\text { Minimal modal logic over } \succeq_{k}^{c} \\
\text { with crisp accessibility relations } \\
\text { over variables } V\end{array}$ & $\mathcal{L}_{B}$ & $\mathrm{~B}_{k}^{c}$ & $\mathbb{M}$ \\
\hline K45 over $€_{k}^{c}$ & $\begin{array}{l}\mathcal{L}_{B} \\
\mathcal{L}_{B} \\
\mathcal{L}_{B}\end{array}$ & 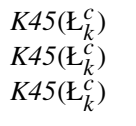 & $\begin{array}{l}\mathbb{M}_{\text {et }} \\
\mathbb{M}_{\text {et }}^{s} \\
\mathbb{M}^{\text {ae }}\end{array}$ \\
\hline KD45 over $Ł_{k}^{c}$ & $\begin{array}{l}\mathcal{L}_{B} \\
\mathcal{L}_{B} \\
\mathcal{L}_{B}\end{array}$ & $\begin{array}{l}K D 45\left(\uplus_{k}^{c}\right) \\
K D 45\left(\bigsqcup_{k}^{c}\right) \\
K D 45\left(\bigsqcup_{k}^{c}\right)\end{array}$ & $\begin{array}{l}\mathbb{M}_{\text {est }} \\
\mathbb{M}_{\text {est }}^{s} \\
\mathbb{M}_{e}^{\text {ae }}\end{array}$ \\
\hline S5 over $€_{k}^{c}$ & $\begin{array}{l}\mathcal{L}_{B} \\
\mathcal{L}_{B} \\
\mathcal{L}_{B}\end{array}$ & 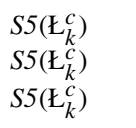 & $\begin{array}{l}\mathbb{M}_{\text {rsyt }} \\
\mathbb{M}_{\text {rsyt }}^{s} \\
\mathbb{M}_{\text {in }}^{\text {ae }}\end{array}$ \\
\hline "Only knowing" over $€_{k}^{c}$ & $\mathcal{L}_{\mathrm{O}}$ & $\mathrm{O}\left(\mathrm{E}_{k}^{c}\right)$ & $\mathbb{M}^{\mathrm{ae}}$ \\
\hline
\end{tabular}

Proof. We only show the case of $K D 45\left(\mathrm{E}_{k}^{c}\right)$. The other cases are obtained by slight adaptations of the proof. By Proposition 2 it is sufficient to show that $\mathbb{M}_{\text {est }}^{s}$ and $\mathbb{M}_{e}^{\text {ae }}$ have the same tautologies.

First suppose there exists an $M=(W, e, R) \in \mathbb{M}_{\text {est }}^{s}$ (i.e. $R=W \times E$ and $\emptyset \neq E \subseteq W$ ) and $w \in W$ such that $\|\phi\|_{M, w}<1$. We show that for $(e(w, \cdot), S) \in \mathbb{M}_{e}^{\text {ae }}$, where $S=\left\{e\left(w^{\prime}, \cdot\right) \mid w^{\prime} \in E\right\}$, we have $\|\phi\|_{(e(w, \cdot), S)}<1$. We will do this by showing that $\|\phi\|_{M, w}=\|\phi\|_{(e(w,), S)}$. To obtain this result we will show by structural induction that for each formula $\gamma$ and for each $z \in W$ we have $\|\gamma\|_{M, z}=\|\gamma\|_{(e(z, \cdot), S)}$. The only notable case is $\gamma=\mathrm{B} \alpha$ for which it holds that $\|\alpha\|_{M, v}=\|\alpha\|_{(e(v, \cdot), S)}$ for all $v \in W$ (induction hypothesis). Now consider $z \in W$, we show that $\|\mathrm{B} \alpha\|_{M, z}=$ $\|\mathrm{B} \alpha\|_{(e(z, \cdot), S)}$.

$$
\begin{aligned}
\|\mathrm{B} \alpha\|_{M, z} & =\inf \left\{\|\alpha\|_{M, v} \mid R(z, v)=1\right\}=\inf \left\{\|\alpha\|_{M, v} \mid v \in E\right\} \\
& =\inf \left\{\|\alpha\|_{(e(v, \cdot), S)} \mid v \in E\right\} \quad=\inf \left\{\|\alpha\|_{\left(v^{\prime}, S\right)} \mid v^{\prime} \in S\right\} \\
& =\|\mathrm{B} \alpha\|_{(e(z, \cdot), S)}
\end{aligned}
$$

where we use the fact that $R=W \times E$ and thus $R(v, z)=1$ iff $v \in E$.

Now to show the other direction. Suppose we have $(v, S) \in \mathbb{M}_{e}^{\mathrm{ae}}$ such that $\|\phi\|_{(v, S)}<1$ and define $M=(W, e, R) \in$ $\mathbb{M}_{\text {est }}^{s}$ as follows: Let $W$ be a set of worlds such that $W$ has the same cardinality as $S^{\prime}=\{v\} \cup S$. Hence there exists a bijection $h: W \rightarrow S^{\prime}: w \rightarrow w^{\prime}$. The mapping $e: W \times V \rightarrow S_{k}$ is defined as $e(w, \cdot)=h(w)$ and we define $R=W \times E$ with $E=\{w \in W \mid h(w) \in S\}$. We show by structural induction that for each formula $\gamma$ and each $w \in W$ we have $\|\gamma\|_{M, w}=\|\gamma\|_{(h(w), S)}$. The only interesting case is $\gamma=\mathrm{B} \alpha$ such that $\|\alpha\|_{M, v}=\|\alpha\|_{(h(v), S)}$ for each $v \in W$ (induction hypothesis). But then we have for $z \in W$ by the induction hypothesis that

$$
\begin{aligned}
\|\mathrm{B} \alpha\|_{M, z} & =\inf \left\{\|\alpha\|_{M, v} \mid R(z, v)=1\right\}=\inf \left\{\|\alpha\|_{M, v} \mid v \in E\right\} \\
& =\inf \left\{\|\alpha\|_{M, v} \mid h(v) \in S\right\} \quad=\inf \left\{\|\alpha\|_{(h(v), S)} \mid h(v) \in S\right\} \\
& =\|\mathrm{B} \alpha\|_{(h(z), S)}
\end{aligned}
$$

In particular it then follows that $\|\phi\|_{M, h^{-1}(v)}=\|\phi\|_{(v, S)}<1$.

To summarize, Table 1 provides a list of the languages and corresponding logics and semantics that have been introduced and used so far.

In [9] the authors characterize stable fuzzy expansions in terms of the above many-valued possible-world semantics. Indeed, the fuzzy belief set induced by an agent's epistemic state $S$, denoted $\mathrm{Bel}_{S}$, is defined in the natural way: for 
each formula $\varphi \in \mathcal{L}_{\mathrm{B}}$, the degree to which the belief in $\varphi$ is supported by $S$ is the truth-degree of $B \varphi$ in $S$, that is, we define

$$
\operatorname{Bel}_{S}(\varphi)=\|\mathrm{B} \varphi\|_{S}=\inf _{w \in S}\|\varphi\|_{(w, S)} .
$$

Moreover, given a set of formulas $A$, a set of propositional evaluations $S_{A}$ is called a fuzzy autoepistemic model of $A$ whenever

$$
S_{A}=\left\{w \in \Omega_{k} \mid\|\phi\|_{\left(w, S_{A}\right)}=1 \text { for each } \phi \in A\right\} .
$$

Intuitively, $S_{A}$ is the set of worlds or "sources" in which all formulas of $A$ are true. Then the following result is proved in [9].

Proposition 4. A fuzzy set of formulas $E: \mathcal{L}_{\mathrm{B}} \rightarrow S_{k}$ is a stable fuzzy expansion of a set of formulas $A$ iff it is the belief set for some fuzzy autoepistemic model $S_{A}$ of A, i.e. $E(\phi)=\|\mathrm{B} \phi\|_{S_{A}}$ for each $\phi \in \mathcal{L}_{\mathrm{B}}$.

Example 4. Here we present an example for fuzzy autoepistemic reasoning in a scenario involving sensor networks. Suppose we have a wireless sensor network consisting of devices that can sense their environment and communicate wirelessly, for instance with the purpose of detecting forest fires in an early stage. We will use fuzzy autoepistemic logic to determine whether there are sensors not working optimally.

Let $t_{i}$ be the variable representing the temperature measured by sensor $i$. Suppose we have an appropriate rescaling to assure that all variables take values in $\Omega_{k}$ and let $e_{i}$ be the variable representing the degree to which sensor $i$ is faulty.

Let us define a new connective $d(\varphi, \psi)^{6}$ as $\neg(\varphi \leftrightarrow \psi)$ for which the semantics is given by the Euclidean distance $\hat{d}$ : for all $x, y \in[0,1], \hat{d}(x, y)=|x-y|$. Then, for $i \neq j$, the formulas

$$
d\left(t_{i}, t_{j}\right) \rightarrow\left(e_{i} \vee e_{j}\right)
$$

capture the idea that if the sensed values of two sensors $t_{i}$ and $t_{j}$ are different, this provides a reason to believe that at least one of those two sensors is faulty. Note that we assume that all sensors are physically close to each other and thus they should measure the same temperature.

On the other hand, the formula

$$
\neg \mathrm{B} e_{i} \rightarrow \neg e_{i}
$$

represents the default information that sensors are not faulty.

As a concrete case, we further assume we are told sensor $e_{2}$ is very reliable, and hence we believe that sensor $e_{2}$ is not faulty $\left(\mathrm{B} \neg e_{2}\right)$, and that the three measured values are $t_{1}^{\prime}=0.2, t_{2}^{\prime}=0.9, t_{3}^{\prime}=0.5$.

The formalization of this scenario then amounts to consider the following theory:

$$
A=\left\{\neg \mathrm{B} e_{1} \rightarrow \neg e_{1}, \neg \mathrm{B} e_{2} \rightarrow \neg e_{2}, \neg \mathrm{B} e_{3} \rightarrow \neg e_{3}, \mathrm{~B} \neg e_{2}, \overline{0.7} \rightarrow\left(e_{1} \vee e_{2}\right), \overline{0.4} \rightarrow\left(e_{2} \vee e_{3}\right), \overline{0.3} \rightarrow\left(e_{1} \vee e_{3}\right)\right\}
$$

Let us see what a stable expansion $E_{A}$ of $A$ must look like.

Suppose there exists a fuzzy autoepistemic model $S$ of $A$. Then by definition it holds that

$$
S=\left\{v \in \Omega_{k} \mid \forall \alpha \in A:\|\alpha\|_{(v, S)}=1\right\} .
$$

For every $v \in S$ it must hold that $\left\|\mathrm{B} \neg e_{2}\right\|_{(v, S)}=1$ and thus that $\inf _{w \in S}\left(1-w\left(e_{2}\right)\right)=1$, i.e. that for all $w \in S$ we have $w\left(e_{2}\right)=0$. Moreover since $\left\|\neg \mathrm{B} e_{i}\right\|_{(v, S)} \leq\left\|\neg e_{i}\right\|_{(v, S)}$ it follows that $1-\left\|\mathrm{B} e_{i}\right\|_{(v, S)} \leq 1-\left\|e_{i}\right\|_{(v, S)}$ and thus $v\left(e_{i}\right) \leq\left\|\mathrm{B} e_{i}\right\|_{(v, S)}=\inf _{w \in S} w\left(e_{i}\right)$. Since for every $v \in S$ we also have $\inf _{w \in S} w\left(e_{i}\right) \leq v\left(e_{i}\right)$, we obtain

$$
v\left(e_{i}\right) \leq \inf _{w \in S} w\left(e_{i}\right) \leq v\left(e_{i}\right)
$$

\footnotetext{
6 The connective $d(\varphi, \psi)$ is well-known in the literature of many-valued logics and it is usually called Chang distance function [12]. The fact that $d$ can be defined in a many-valued logical setting is a peculiarity of MV-algebras and also for this reason we believe these structures to be a suitable algebraic setting.
} 
for $i \in\{1,2,3\}$. If there exist $v_{1}, v_{2} \in S$, then we have that $v_{1}\left(e_{i}\right)=\inf _{w \in S} w\left(e_{i}\right)=v_{2}\left(e_{i}\right)$ for all $i \in\{1,2,3\}$, it follows that $S=\{v\}$ is a singleton such that $v\left(e_{2}\right)=0$. Taking into account the remaining formulas in $A$ we obtain that it must hold that $0.7 \leq v\left(e_{1}\right), 0.4 \leq v\left(e_{3}\right)$ and $0.3 \leq \max \left(v\left(e_{1}\right), v\left(e_{3}\right)\right)$. Actually, we can check that $v$ is the minimal element in $\Omega_{k}$ such that these inequalities are satisfied, i.e. $v\left(e_{1}\right)=0.7, v\left(e_{2}\right)=0$ and $v\left(e_{3}\right)=0.4$. Indeed, let $z \in \Omega_{k}$ such that $z \leq v$ and such that $z$ satisfies the above inequalities. Then we obtain $z\left(e_{i}\right) \leq v\left(e_{i}\right)=\inf _{w \in S} w\left(e_{i}\right)$ and thus $\left\|\neg \mathrm{B} e_{i} \rightarrow \neg e_{i}\right\|_{(z, S)}=\left\|e_{i} \rightarrow \mathrm{B} e_{i}\right\|_{(z, S)}=1$ for $i \in\{1,2,3\}$. This implies that $z \in S$, a contradiction if $z \neq v$. Hence if there exists a fuzzy autoepistemic model $S$ of $A$ it has to be

$$
S=\left\{v \in \Omega_{k} \mid v\left(e_{1}\right)=0.7, v\left(e_{2}\right)=0, v\left(e_{3}\right)=0.4\right\} .
$$

Using the definition one can now easily show that $S$ is indeed a fuzzy autoepistemic model of $A$. In particular, it has to be shown that

$$
\left\{v \in \Omega_{k} \mid v\left(e_{1}\right)=0.7, v\left(e_{2}\right)=0, v\left(e_{3}\right)=0.4\right\}=\left\{w \in \Omega_{k} \mid \forall \alpha \in A:\|\alpha\|_{(w, S)}=1\right\},
$$

or in other words, if $w \neq v$, then $\|\alpha\|_{(w, S)}<1$ for some $\alpha \in A$. The corresponding stable expansion $E_{A}: \mathcal{L}_{\mathrm{B}} \rightarrow S_{k}$ is such that $E_{A}\left(\mathrm{~B} e_{1}\right)=E_{A}\left(e_{1}\right)=0.7, E_{A}\left(\mathrm{~B} e_{2}\right)=E_{A}\left(e_{2}\right)=0$ and $E_{A}\left(\mathrm{~B} e_{3}\right)=E_{A}\left(e_{3}\right)=0.4$.

\subsection{Fuzzy stable sets}

On the other hand, as in the classical case, we can also characterize fuzzy belief sets, and a forteriori stable fuzzy expansions, by the following graded version of syntactical Stalnaker's notion of stable set of formulas (cf. [20]).

Definition 2. Let $\Gamma: \mathcal{L}_{\mathrm{B}} \rightarrow S_{k}$ be a fuzzy set of formulas and let $\hat{\Gamma}=\left\{\overline{\Gamma(\varphi)} \rightarrow \varphi^{*} \mid \varphi \in \mathcal{L}_{B}\right\}$. We say that $\Gamma$ is a fuzzy stable set if the following conditions hold:

(1) $\hat{\Gamma}$ is propositionally consistent, i.e. $\hat{\Gamma} \nvdash \overline{0}$.

(2) If $\hat{\Gamma} \vdash \bar{c} \rightarrow \varphi^{*}$, then $\Gamma(\varphi) \geq c$.

(3) $\Gamma(\varphi)=\Gamma(\mathrm{B} \varphi)$.

(4) $1-\Gamma(\varphi)=\Gamma(\neg \mathrm{B} \varphi)$.

The conditions (1)-(4) in Definition 2 are the graded counterparts of the classical ones in [20]. In particular, condition (3) expresses that fuzzy stable sets are closed by the belief operator B, i.e. $\varphi$ belongs to $\Gamma$ as much as $\mathrm{B} \varphi$ does. On the other hand condition (4) expresses a kind of graded closed world assumption for fuzzy stable sets: $\mathrm{B} \varphi$ is not believed (in the sense of belonging to $\Gamma$ ) iff $\varphi$ is not in the set $\Gamma$.

Proposition 5. $\Gamma$ is a fuzzy stable set iff $\Gamma$ is a fuzzy belief set for some non-empty epistemic state. In other words, $\Gamma$ is a fuzzy stable set iff $\Gamma=\mathrm{Bel}_{S}$ for some $S \neq \emptyset$.

\section{Proof.}

$(\Leftarrow)$ First we show that a fuzzy belief set $\Gamma$ is a fuzzy stable set. By definition of fuzzy belief set we know that there exists a $\emptyset \neq S \subseteq \Omega_{k}$ such that $\Gamma(\varphi)=\|\mathrm{B} \varphi\|_{S}$ for each formula $\varphi$. In order to show that $\hat{\Gamma}$ is propositionally consistent, by the strong completeness of $\mathrm{七}_{k}^{c}$, it is sufficient to show that there exists a $v \in \Omega_{k}^{*}$ such that for each formula $\varphi$ we have $\Gamma(\varphi) \leq v\left(\varphi^{*}\right)$. Indeed, it then follows that for each $\varphi$ we have $v\left(\overline{\Gamma(\varphi)} \rightarrow \varphi^{*}\right)=1$ and thus $v(\alpha)=1$ for all $\alpha \in \hat{\Gamma}$. Let $w \in S$ be arbitrary but fixed and define $v$ such that $v\left(\varphi^{*}\right)=\|\varphi\|_{(w, S)}$ for each $\varphi$. It follows that

$$
\Gamma(\varphi)=\inf _{z \in S}\|\varphi\|_{(z, S)} \leq v\left(\varphi^{*}\right)
$$

which proves (1). Next, assume that $\hat{\Gamma} \vdash \bar{c} \rightarrow \varphi^{*}$, or by strong completeness of $€_{k}^{c}$ that $\hat{\Gamma} \models \bar{c} \rightarrow \varphi^{*}$. We show that $\Gamma(\varphi) \geq c$. Note, similar as above, that for each $w \in S$ we have that $v$ with $v\left(\psi^{*}\right)=\|\psi\|_{(w, S)}$ for all $\psi \in \mathcal{L}_{B}$ is a model of $\hat{\Gamma}$ and hence of $\bar{c} \rightarrow \varphi^{*}$. Therefore $c \leq\|\varphi\|_{(w, S)}$ for each $w \in S$ and $c \leq \inf _{w \in S}\|\varphi\|_{(w, S)}=\Gamma(\varphi)$ which proves (2). Proving (3) follows easily by noting that 


$$
\Gamma(\varphi)=\|\mathrm{B} \varphi\|_{S}=\|\mathrm{BB} \varphi\|_{S}=\Gamma(\mathrm{B} \varphi) .
$$

Finally, to show (4), observe that $\Gamma(\neg \mathrm{B} \varphi)$ equals

$$
\|\mathrm{B} \neg \mathrm{B} \varphi\|_{S}=\inf _{w \in S}\|\neg \mathrm{B} \varphi\|_{(w, S)}=\inf _{w \in S}\left(1-\|\mathrm{B} \varphi\|_{(w, S)}\right)=\inf _{w \in S}(1-\Gamma(\varphi))
$$

which is equal to $1-\Gamma(\varphi)$.

$\Leftrightarrow)$ Now, let $\Gamma$ be a fuzzy stable set. Define

$$
S^{*}=\left\{u \in \Omega_{k}^{*} \mid u(\alpha)=1, \forall \alpha \in \hat{\Gamma}\right\} .
$$

Note that $S^{*}$ is nonempty by (1). We show that for each $\varphi$ and $S=\left\{u_{\mid V} \mid u \in S^{*}\right\} \neq \emptyset^{7}$ we have

$$
\Gamma(\varphi)=\|\mathrm{B} \varphi\|_{S} .
$$

First, note that for a formula $\varphi$ we have by definition of $S^{*}$ that $w\left(\overline{\Gamma(\mathrm{B} \varphi)} \rightarrow(\mathrm{B} \varphi)^{*}\right)=1$, or $w\left((\mathrm{~B} \varphi)^{*}\right) \geq \Gamma(\mathrm{B} \varphi)$, for each $w \in S^{*}$. By (3) it then follows that $w\left((\mathrm{~B} \varphi)^{*}\right) \geq \Gamma(\mathrm{B} \varphi)=\Gamma(\varphi)$ for each $w \in S^{*}$. We show that also $w\left((\mathrm{~B} \varphi)^{*}\right) \leq \Gamma(\varphi)$ from which it then follows that

$$
w\left((\mathrm{~B} \varphi)^{*}\right)=\Gamma(\varphi)
$$

for each $w \in S^{*}$. Indeed, since $\overline{\Gamma(\neg \mathrm{B} \varphi)} \rightarrow(\neg \mathrm{B} \varphi)^{*}$ is in $\hat{\Gamma}$ and thus $\Gamma(\neg \mathrm{B} \varphi) \leq w\left((\neg \mathrm{B} \varphi)^{*}\right)=w\left(\neg(\mathrm{B} \varphi)^{*}\right)$ we have

$$
w\left((\mathrm{~B} \varphi)^{*}\right)=1-w\left(\neg(\mathrm{B} \varphi)^{*}\right) \leq 1-\Gamma(\neg \mathrm{B} \varphi) .
$$

Hence by (4) it follows that $w\left((\mathrm{~B} \varphi)^{*}\right) \leq \Gamma(\varphi)$. We will now use the fact that

$$
w\left((\mathrm{~B} \varphi)^{*}\right)=\Gamma(\varphi)
$$

for each $w \in S^{*}$ to show that for each formula $\alpha$ and each $w \in S^{*}$ we have $w\left(\alpha^{*}\right)=\|\alpha\|_{\left(w_{\mid V}, S\right)}$ from which we can conclude that

$$
\Gamma(\varphi)=w\left((\mathrm{~B} \varphi)^{*}\right)=\|\mathrm{B} \varphi\|_{\left(w_{\mid V}, S\right)}=\|\mathrm{B} \varphi\|_{S}
$$

for each formula $\varphi$ and an arbitrary $w \in S^{*}$. We show $w\left(\alpha^{*}\right)=\|\alpha\|_{\left(w_{\mid V}, S\right)}$ by structural induction. The only notable case is $\alpha=\mathrm{B} \psi$. By the definition of $S^{*}$ we have $\Gamma(\psi) \leq u\left(\psi^{*}\right)$ for all $u \in S^{*}$ and hence

$$
\Gamma(\psi) \leq \inf _{u \in S^{*}} u\left(\psi^{*}\right) .
$$

Now suppose that $\Gamma(\psi)<\inf _{u \in S^{*}} u\left(\psi^{*}\right)$, i.e. for each $u \in S^{*}$ we have $\Gamma(\psi)<u\left(\psi^{*}\right)$. Since the set of truth values is finite there exists a $v \in S^{*}$ such that $v\left(\psi^{*}\right)=\min _{u \in S^{*}} u\left(\psi^{*}\right)$. Hence for all $u \in S^{*}$ we have $v\left(\psi^{*}\right) \leq$ $u\left(\psi^{*}\right)$ and thus $u\left(\overline{v\left(\psi^{*}\right)} \rightarrow \psi^{*}\right)=1$. By the strong completeness of $\mathfrak{七}_{k}^{c}$ and the definition of $S^{*}$ it follows that

$$
\hat{\Gamma} \vdash \overline{v\left(\psi^{*}\right)} \rightarrow \psi^{*} .
$$

By (2), it then follows that $\Gamma(\psi) \geq v\left(\psi^{*}\right)$. Since $v \in S^{*}$ it follows that $\Gamma(\psi)<v\left(\psi^{*}\right)$ and thus that $\Gamma(\psi)>$ $\Gamma(\psi)$, a contradiction. Hence $\Gamma(\psi)=\inf _{u \in S^{*}} u\left(\psi^{*}\right)$ and by the induction hypothesis we conclude that

$$
\begin{aligned}
w\left(\alpha^{*}\right) & =w\left((\mathrm{~B} \psi)^{*}\right)=\Gamma(\psi)=\inf _{u \in S^{*}} u\left(\psi^{*}\right) \\
& =\inf _{u \in S^{*}}\|\psi\|_{\left(u_{\mid V}, S\right)}=\inf _{z \in S}\|\psi\|_{(z, S)}=\|\alpha\|_{\left(w_{\mid V}, S\right)}
\end{aligned}
$$

By combining Propositions 4 and 5, we can easily derive the following list of properties that fuzzy stable expansions satisfy. Note that it would be more difficult to check these properties directly from the definition of fuzzy stable expansions.

\footnotetext{
${ }^{7} u_{\mid V}$ is the function $u_{\mid V}: V \rightarrow S_{k}$.
} 
Proposition 6. Let $A$ be a set of $\mathcal{L}_{B}$-formulas. If $E_{A}$ is fuzzy stable expansion of $A$, then the following properties hold:

(i) $\left\{\phi \in \mathcal{L}_{\mathrm{B}} \mid A^{*} \vdash \phi^{*}\right\} \subseteq \underline{E_{A}}$;

(ii) $\bar{r} \rightarrow \phi \in E_{A}$ iff $E_{A}(\phi) \overline{\geq}$;

(iii) If $\bar{r} \rightarrow \phi \overline{\in E_{A}}$ and $\bar{s} \rightarrow \phi \notin \underline{E_{A}}$ for $s>r$, then $E_{A}(\phi)=r$;

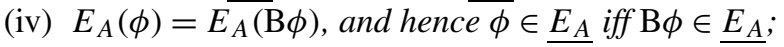

(v) $1-E_{A}(\phi)=E_{A}(\neg \mathrm{B} \phi)$.

Proof. First of all, by Propositions 4 and 5 it follows that $E_{A}$ is a fuzzy stable set. Then, (i) is the trivial consequence of $E_{A}$ being (propositionally) deductively closed and containing $A$, (ii) and (iii) directly follows from the definition of $\overline{E_{A}}$, while (iv) and (v) are just translations from analogous properties of fuzzy stable sets from Proposition 5.

This last proposition confirms that many natural properties that one would expect to hold for fuzzy stable expansions are indeed satisfied. Firstly, (i) shows that all propositional consequences of the initial beliefs in $A$ will belong (to degree 1) to any fuzzy stable expansion of $A$, i.e. $E_{A}$ contains at least all the logical consequences of $A$. Properties (ii) and (iii) state that an agent fully believes a formula of the kind $\bar{r} \rightarrow \phi$ iff he/she believes $\phi$ at least to degree $r$ and if he/she does not fully believe $\bar{s} \rightarrow \phi$ to a higher degree $s$, then the degree of belief in $\phi$ should be exactly $r$. Finally, (iv) and (v) state that beliefs are closed by iterating B and by a closed world assumption principle.

\section{6. “Only knowing” operators and stable fuzzy expansions}

Let us extend $\mathcal{L}_{\mathrm{B}}$ with a modal operator $\mathrm{O}$ where a formula $\mathrm{O} \psi$ will be interpreted as " $\psi$ is all that is believed". We denote this language by $\mathcal{L}_{\mathrm{O}}$. In the classical case [6], the semantics for a corresponding logic is defined as follows. Given an epistemic state $S$ consisting of a set of classical evaluations, a formula $\mathrm{O} \psi$ is true in $S$ whenever $\psi$ is true in any structure $(z, S)$ with $z \in S$, and false in any structure $\left(z^{\prime}, S\right)$ with $z^{\prime} \notin S$.

We can straightforwardly generalize this condition to the many-valued case by defining

$$
\|\mathrm{O} \psi\|_{(w, S)}=\min \left(\inf _{z \in S}\|\psi\|_{(z, S)}, \inf _{z \notin S}\|\neg \psi\|_{(z, S)}\right),
$$

where now $w \in \Omega_{k}$ and $S \subseteq \Omega_{k}$. Other formulas are evaluated as in fuzzy autoepistemic logic. If we then add another modal operator $\mathrm{N}$ whose truth evaluation in a pair $(w, S)$ is

$$
\|\mathrm{N} \psi\|_{(w, S)}=\inf _{z \notin S}\|\psi\|_{(z, S)},
$$

then it is easy to see that the semantics of $\mathrm{O} \psi$ is exactly that of $\mathrm{B} \psi \wedge \mathrm{N} \neg \psi$. Notice that $\|\mathrm{N} \psi\|_{(w, S)}=\|\mathrm{B} \psi\|_{\left(w, \Omega_{k} \backslash S\right)}$. Hence by Theorem 4 it follows that $\mathrm{N}$ is another K45 "operator". Again, since the truth value of $\mathrm{O} \psi$ (and $\mathrm{N} \psi$ ) in a structure $(w, S)$ does not depend on $w$, we will also write $\|\mathrm{O} \psi\|_{S}$ and $\|\mathrm{N} \psi\|_{S}$ to denote $\|\mathrm{O} \psi\|_{(w, S)}$ and $\|\mathrm{N} \psi\|_{(w, S)}$ respectively.

There is an intuitive link between the operator $\mathrm{N}$ and the guaranteed possibility function of [23]. However, there are some clear differences as well. Here we consider crisp accessibility relations over graded worlds, whereas [23] considers graded accessibility relations (encoded by possibility distributions) between Boolean, i.e. classical, worlds. The fact that only knowing can be understood as the conjunction of belief (necessity) and guaranteed possibility was already pointed out in [24] in a classical context.

To summarize, the language $\mathcal{L}_{\mathrm{O}}$ we consider is built from propositional variables in $V$, truth constants $\left\{\bar{r} \mid r \in S_{k}\right\}$, the connectives from Łukasiewicz logic and modal operators B, N and $\mathrm{O}$. We evaluate formulas using the class of semantical structures $\mathbb{M}^{\text {ae }}=\left\{(w, S) \mid w \in \Omega_{k}, S \subseteq \Omega_{k}\right\}$. The truth of a formula $\phi \in \mathcal{L}_{\mathrm{O}}$ relative to a structure $(w, S)$ is inductively defined as follows:

- If $\phi$ is a propositional formula from $\mathcal{L}_{\mathrm{O}}$, then $\|\phi\|_{(w, S)}=w(\phi)$.

- If $\phi$ is a truth constant $\bar{c}$, then $\|\phi\|_{(w, S)}=c$.

- If $\phi=\mathrm{B} \psi$, then $\|\phi\|_{(w, S)}=\inf \left\{\|\psi\|_{(v, S)} \mid v \in S\right\}$.

- If $\phi=\mathrm{N} \psi$, then $\|\phi\|_{(w, S)}=\inf \left\{\|\psi\|_{(v, S)} \mid v \notin S\right\}$.

- If $\phi=\psi \rightarrow \gamma$, then $\|\phi\|_{(w, S)}=\left(\|\psi\|_{(w, S)} \Rightarrow\|\gamma\|_{(w, S)}\right)$. 
Note that we actually use a partition of the worlds in two subsets: the set $S$ is used for the semantics of B and its complement $\Omega_{k} \backslash S$ for the semantics of $\mathrm{N}$.

As in the classical case [6], we will provide a sound and complete axiomatization for the fuzzy logic of "only knowing", which we will denote as $\mathrm{O}\left(\mathfrak{七}_{k}^{c}\right)$. Specifically, $\mathrm{O}\left(\mathfrak{七}_{k}^{c}\right)$ has the following axioms:

(i) axioms of $\mathfrak{七}_{k}^{c}$,

(ii) axioms of $K 45\left(\mathrm{七}_{k}^{c}\right)$ for both $\mathrm{B}$ and $\mathrm{N}$,

(iii) $\phi \rightarrow \mathrm{B} \phi$, where all variables and constants in $\phi$ occur in the scope of an operator $\mathrm{N}$ or $\mathrm{B}$,

(iv) $\phi \rightarrow \mathrm{N} \phi$, where all variables and constants in $\phi$ occur in the scope of an operator $\mathrm{N}$ or $\mathrm{B}$,

(v) $\neg \mathrm{B} \phi \vee \neg \mathrm{N} \phi$, if $\neg \phi$ is satisfiable and does not contain any modal operator,

(vi) $\mathrm{O} \phi \leftrightarrow(\mathrm{B} \phi \wedge \mathrm{N} \neg \phi)$,

and the following inference rules: modus ponens and necessitation rules for $\mathrm{N}$ and $\mathrm{B}$.

Axioms (iii)-(vi) are many-valued versions of the ones proposed by Levesque in [6]. For axioms (iii) and (iv), the idea is to capture the fact that the truth value of a truth constant or any formula $\varphi$ starting by a modality B or $\mathrm{N}$ in a model $(w, S)$ depends only on $S$ and not on the particular interpretation $w$. Hence, that value will also coincide with the value of $\mathrm{B} \varphi$ or $\mathrm{N} \varphi$, since they are the infimum of identical values. In other words, together with axiom (4), they specify that we can avoid nested beliefs (in the general sense of $\mathrm{B}, \mathrm{N}$ and $\mathrm{O}$ ). The intuition behind axiom (v) is the following. Since a propositional formula $\phi$ that is not a tautology cannot be true to degree 1 in all possible worlds, an agent cannot believe $\phi$ in a given epistemic state $S$ and believe $\phi$ in the complementary epistemic state $\Omega_{k} \backslash S$. Actually one can check that in our fuzzy framework all the above axioms are tautologies and the rules preserve tautologies in every structure $(w, S) \in \mathbb{M}^{\mathrm{ae}}$. In particular, we have the following result.

Lemma 5. The axioms (i)-(vi) are sound w.r.t. the class of structures $\mathbb{M}^{\text {ae }}$.

Proof. Axioms (i) and (ii) follow from previous results.

Axioms (iii) and (iv) are easy to check. It only has to be shown that these axioms are tautologies for formulas of the form $\phi=\mathrm{B} \alpha$ and $\phi=\mathrm{N} \alpha$. Indeed by truth functionality we then obtain that these axioms are tautologies for formulas where all variables and constants occur in the scope of an operator $\mathrm{N}$ or $\mathrm{B}$. For axiom (iii), $(w, S) \in \mathbb{M}^{\mathrm{ae}}$ and $\phi=\mathrm{B} \alpha$ we have

$$
\|\mathrm{B} \phi\|_{(w, S)}=\inf _{v \in S}\|\mathrm{~B} \alpha\|_{(v, S)}=\inf _{z \in S}\|\alpha\|_{(z, S)}=\|\phi\|_{(w, S)} .
$$

The other cases follow similarly.

For axiom (v), suppose $\neg \phi$ is satisfiable, i.e. there exists $w^{*} \in \Omega_{k}^{*}$ such that $w^{*}(\phi)=0$. For a structure $(w, S) \in \mathbb{M}^{\text {ae }}$ we then have $\|\neg \mathrm{B} \phi \vee \neg \mathrm{N} \phi\|_{(w, S)}=1$ iff $\max \left(\|\neg \mathrm{B} \phi\|_{(w, S)},\|\neg \mathrm{N} \phi\|_{(w, S)}\right)=1$ iff $\|\mathrm{B} \phi\|_{(w, S)}=0$ or $\|\mathrm{N} \phi\|_{(w, S)}=0$ iff there exists $z \in S$ such that $z(\phi)=\|\phi\|_{(z, S)}=0$ or there exists $z \notin S$ such that $z(\phi)=\|\phi\|_{(z, S)}=0$. This is satisfied by the fact that there exists $w^{\prime} \in \Omega_{k}=S \cup\left(\Omega_{k} \backslash S\right)$ such that $w^{\prime}(\phi)=0$. Indeed let $w^{\prime}=w_{\mid V}^{*}$.

Similarly as in Section 3, we can show that there exists a reduction of the satisfiability problem for the fuzzy logic of only knowing to the classical counterpart. For every structure $(w, S) \in \mathbb{M}^{\text {ae }}$ we then have $\|\Delta(\phi \leftrightarrow \bar{r})\|_{(w, S)}=1$ iff $\|\phi\|_{(w, S)}=r$. Define $\mathcal{L}_{\mathrm{O}}^{\prime} \subseteq \mathcal{L}_{\mathrm{O}}$ as the set of formulas built from atomic propositions of the form $\{\Delta(p \leftrightarrow \bar{r}) \mid p \in V$, $\left.r \in S_{k}\right\}$, Łukasiewicz connectives and operators $\mathrm{B}, \mathrm{N}$ and $\mathrm{O}$ :

- $\Delta(p \leftrightarrow \bar{r}) \in \mathcal{L}_{\mathrm{O}}^{\prime}$ for every $p \in V$ and $r \in S_{k}$

$-(\phi \rightarrow \psi) \in \mathcal{L}_{\mathrm{O}}^{\prime}$ if $\phi, \psi \in \mathcal{L}_{\mathrm{O}}^{\prime}$

- $\mathrm{B} \phi \in \mathcal{L}_{\mathrm{O}}^{\prime}$ if $\phi \in \mathcal{L}_{\mathrm{O}}^{\prime}$

- $\mathrm{N} \phi \in \mathcal{L}_{\mathrm{O}}^{\prime}$ if $\phi \in \mathcal{L}_{\mathrm{O}}^{\prime}$

- $\mathrm{O} \phi \in \mathcal{L}_{\mathrm{O}}^{\prime}$ if $\phi \in \mathcal{L}_{\mathrm{O}}^{\prime}$

Lemma 6. Given a formula $\phi \in \mathcal{L}_{\mathrm{O}}$ and a truth value $r \in S_{k}$, there exists a formula $\left(\phi_{r}\right)^{\prime} \in \mathcal{L}_{\mathrm{O}}^{\prime}$ such that for each structure $(w, S) \in \mathbb{M}^{\text {ae }}$ it holds that 


$$
\|\phi\|_{(w, S)}=r \text { iff }\left\|\left(\phi_{r}\right)^{\prime}\right\|_{(w, S)}=1,
$$

where $\phi_{r}$ is a short notation for the formula $\Delta(\phi \leftrightarrow \bar{r})$ with $\phi \in \mathcal{L}_{\mathrm{O}}$.

Proof. This lemma can be shown in a similar way as in the proof of Lemma 3. In particular the claim now also has to be checked for formulas of the form $\mathrm{N} \phi$ which can be done entirely analogously as for formulas of the form $\mathrm{B} \phi$.

We will use this lemma together with the following proposition to show that the proposed axiomatization is complete w.r.t. the proposed semantics. In Proposition 7 and Theorem 5, besides the languages $\mathcal{L}_{\mathrm{O}}$ and $\mathcal{L}_{\mathrm{O}}^{\prime}$ and structures $\mathbb{M}^{\mathrm{ae}}$, we also consider the following languages:

- $\mathcal{L}_{\mathrm{O}}^{+}$, which is an extension of $\mathcal{L}_{\mathrm{O}}$ with an additional set of variables $\hat{V}=\left\{p_{r} \mid p \in V, r \in S_{k}\right\}$, i.e. the language built from variables $V \cup \hat{V}$, Łukasiewicz connectives and operators $\mathrm{B}, \mathrm{N}$ and $\mathrm{O}$;

- $\mathcal{L}_{\mathrm{O}}^{C}$, built from variables $\hat{V}$, classical connectives $(\wedge, \vee, \neg, \rightarrow)$ and operators $\mathrm{B}, \mathrm{N}$ and $\mathrm{O}$;

and the following classes of semantic structures:

- $\mathbb{M}_{+}^{\mathrm{ae}}=\left\{(w, S) \mid w \in \Omega_{k}^{+}, S \subseteq \Omega_{k}^{+}\right\}$where $\Omega_{k}^{+}=\left\{w \mid w: V \cup \hat{V} \rightarrow S_{k}\right\}$;

- $\mathbb{M}_{B}^{\mathrm{ae}}=\left\{(w, S) \mid w \in \Omega_{k}^{B}, S \subseteq \Omega_{k}^{B}\right\}$ where $\Omega_{k}^{B}=\{w \mid w: \hat{V} \rightarrow\{0,1\}\}$.

Proposition 7. Suppose $\phi \in \mathcal{L}_{\mathrm{O}}^{\prime}$ is a tautology w.r.t. $\mathbb{M}^{\mathrm{ae}}$, then $\phi$ is provable in $\mathrm{O}\left(\ell_{k}^{c}\right)$.

Proof. Suppose $\phi \in \mathcal{L}_{\mathrm{O}}^{\prime}$ is a tautology in $\mathbb{M}^{\mathrm{ae}}$. Define $\phi^{\prime \prime}$ as the formula in $\mathcal{L}_{O}^{+}$obtained by replacing each subformula $\Delta(p \leftrightarrow \bar{r})$ by the atom $p_{r} \in \hat{V}$. We show that $\Psi^{\prime \prime} \rightarrow \phi^{\prime \prime}$, with

$$
\Psi^{\prime \prime}=\Delta\left[\bigwedge_{p \in V, r \in S_{k}}\left(\Delta(p \leftrightarrow \bar{r}) \leftrightarrow p_{r}\right)\right],
$$

is a tautology in $\mathbb{M}_{+}^{\mathrm{ae}}$. Indeed, consider a structure $(w, S)$ in $\mathbb{M}_{+}^{\mathrm{ae}}$. Note that $\left\|\Psi^{\prime \prime}\right\|_{(w, S)}=1$ if $\|\Delta(p \leftrightarrow \bar{r})\|_{(w, S)}=$ $\left\|p_{r}\right\|_{(w, S)}$ for all $p \in V$ and $\left\|\Psi^{\prime \prime}\right\|_{(w, S)}=0$ otherwise. If $\left\|\Psi^{\prime \prime}\right\|_{(w, S)}=1$, then $\left\|\phi^{\prime \prime}\right\|_{(w, S)}=1$ since $\phi$ is a tautology in $\mathbb{M}^{\text {ae }}$ and in this case we have $\|\Delta(p \leftrightarrow \bar{r})\|_{(w, S)}=\left\|p_{r}\right\|_{(w, S)}$ for all $p \in V$. If $\left\|\Psi^{\prime \prime}\right\|_{(w, S)}=0$, then trivially $\left\|\Psi^{\prime \prime}\right\|_{(w, S)} \leq\left\|\phi^{\prime \prime}\right\|_{(w, S)}$. Hence, in both cases we obtain $\left\|\Psi^{\prime \prime} \rightarrow \phi^{\prime \prime}\right\|_{(w, S)}=1$.

Next we show that $\Sigma^{\prime \prime} \rightarrow \phi^{\prime \prime}$, with

$$
\Sigma^{\prime \prime}=\bigwedge_{p \in V}\left(\bigvee_{r \in S_{k}} p_{r} \wedge \bigwedge_{s, t \in S_{k}, s \neq t} \neg\left(p_{r} \wedge p_{s}\right)\right),
$$

is a tautology in $\mathbb{M}_{B}^{\text {ae }}$. Indeed, consider a structure $(\hat{w}, \hat{S})$ in $\mathbb{M}_{B}^{\text {ae }}$. We show that $\left\|\Sigma^{\prime \prime}\right\|_{(\hat{w}, \hat{S})}=1$ implies $\left\|\phi^{\prime \prime}\right\|_{(\hat{w}, \hat{S})}=1$. Suppose that $\left\|\Sigma^{\prime \prime}\right\|_{(\hat{w}, \hat{S})}=1$. By the definition of $\Sigma^{\prime \prime}$, it then holds that for each $p \in V$ there exists exactly one $r_{p} \in S_{k}$ such that $\hat{w}\left(p_{r_{p}}\right)=\left\|p_{r_{p}}\right\|_{(\hat{w}, \hat{S})}=1$. We will now show that there exists a $(w, S) \in \mathbb{M}_{+}^{\text {ae }}$ such that $\left\|\phi^{\prime \prime}\right\|_{(\hat{w}, \hat{S})}=$ $\left\|\phi^{\prime \prime}\right\|_{(w, S)}$ and such that $\left\|\Psi^{\prime \prime}\right\|_{(w, S)}=1$. Since $\Psi^{\prime \prime} \rightarrow \phi^{\prime \prime}$ is a tautology in $\mathbb{M}_{+}^{\text {ae }}$ we then obtain that $\left\|\phi^{\prime \prime}\right\|_{(\hat{w}, \hat{S})}=1$. To do this, we first define a mapping $f: \Omega_{k}^{B} \rightarrow \Omega_{k}^{+}$as follows. For $\hat{v} \in \Omega_{k}^{B}$, let $f(\hat{v}): V \cup \hat{V} \rightarrow S_{k}$ be the evaluation such that $(f(\hat{v}))\left(p_{r}\right)=\hat{v}\left(p_{r}\right)$ for all $p_{r} \in \hat{V}$ and for each $p \in V$ let $(f(\hat{v})(p))=r_{p}$ where $r_{p}$ is the unique element in $S_{k}$ such that $\hat{v}\left(p_{r_{p}}\right)=\left\|p_{r_{p}}\right\|_{(\hat{v}, \hat{S})}=1$. We define $(w, S) \in \mathbb{M}_{+}^{\text {ae }}$ as follows: $w=f(\hat{w})$ and $S=\{f(\hat{v}) \mid \hat{v} \in \hat{S}\}$. It then holds that $\left\|\Psi^{\prime \prime}\right\|_{(w, S)}=1$ and $\left\|\phi^{\prime \prime}\right\|_{(\hat{w}, \hat{S})}=\left\|\phi^{\prime \prime}\right\|_{(w, S)}$.

Since $\Sigma^{\prime \prime} \rightarrow \phi^{\prime \prime}$ is a tautology in $\mathbb{M}_{B}^{\text {ae }}$ and the classical logic of only knowing is sound and complete w.r.t. $\mathbb{M}_{B}^{\text {ae }}$ [6], there is a corresponding proof $\Gamma$ for $\Sigma^{\prime \prime} \rightarrow \phi^{\prime \prime}$. We will transform this proof to a proof for $\phi^{\prime}$ in $\mathrm{O}\left(\mathfrak{七}_{k}^{c}\right)$. First note that $\Sigma^{\prime}$, which is obtained from $\Sigma^{\prime \prime}$ by replacing each $p_{r}$ by $\Delta(p \leftrightarrow \bar{r})$, is a theorem in $\mathfrak{七}_{k}^{c}$ and hence also a theorem in $\mathrm{O}\left(\mathfrak{七}_{k}^{c}\right)$. By modus ponens, it is now sufficient to show that $\Sigma^{\prime} \rightarrow \phi^{\prime}$ is a theorem as well. This follows trivially by substituting in every formula in $\Gamma$ expressions of the form $p_{r}$ by $\Delta(p \leftrightarrow \bar{r})$. 
In the following theorem we will show completeness for $\mathrm{O}\left(\mathfrak{七}_{k}^{c}\right)$ w.r.t. $\mathbb{M}^{\mathrm{ae}}$.

Theorem 5. Every tautology in $\mathbb{M}^{\text {ae }}$ is a theorem in $\mathrm{O}\left(t_{k}^{c}\right)$.

Proof. Suppose $\phi$ is a tautology in $\mathbb{M}^{\text {ae }}$. Trivially, $\phi_{1}=\Delta \phi$ is a tautology as well and by Lemma 6 , it follows that $\left(\phi_{1}\right)^{\prime} \in \mathcal{L}_{\mathrm{O}}^{\prime}$ constructed analogously as in Lemma 3 is also a tautology. By Proposition 7 it then follows that there is a proof for $\left(\phi_{1}\right)^{\prime}$ in $\mathrm{O}\left(\mathrm{E}_{k}^{c}\right)$. We will now show that this implies that there also exists a proof for $\phi$ in $\mathrm{O}\left(\mathrm{E}_{k}^{c}\right)$. We will do this by showing that for each $\varphi \in \mathcal{L}_{\mathrm{O}}$ and for each $r \in S_{k}$ it holds that $\varphi_{r}=\Delta(\varphi \leftrightarrow \bar{r})$ is provably equivalent to $\left(\varphi_{r}\right)^{\prime}$ as constructed in Lemma 3, i.e. that $\mathrm{O}\left(\mathfrak{\leftarrow}_{k}^{c}\right)$ proves $\varphi_{r} \leftrightarrow\left(\varphi_{r}\right)^{\prime}$. In particular, this implies that $\left(\phi_{1}\right)^{\prime}$ and $\phi_{1}=\Delta \phi$ are provable equivalent formulas. Since by Proposition $7,\left(\phi_{1}\right)^{\prime}$ is a theorem in $\mathrm{O}\left(\mathrm{七}_{k}^{c}\right)$ it then follows that $\Delta \phi$ and hence $\phi$ is a theorem as well.

Let us show by induction that for each $\varphi \in \mathcal{L}_{\mathrm{O}}$ and for each $r \in S_{k}$ it holds that $\varphi_{r}=\Delta(\varphi \leftrightarrow \bar{r})$ is provably equivalent to $\left(\varphi_{r}\right)^{\prime}$. The only non-trivial step in this proof is to show that there exists a $\left(\mathrm{B} \alpha_{r}\right)^{\prime} \in \mathcal{L}_{\mathrm{O}}$ provably equivalent to $\Delta(\mathrm{B} \alpha \leftrightarrow \bar{r})$ given that the claim holds for $\alpha$. The case of $\mathrm{N}$ can be proved analogously. First note that if $r<1$, $\mathrm{E}_{k}^{c}$ proves that $\Delta(\mathrm{B} \alpha \leftrightarrow \bar{r})$ is equivalent to

$$
\left(\bigvee_{t \geq r} \Delta(\mathrm{B} \alpha \leftrightarrow \bar{t})\right) \wedge\left(\neg \bigvee_{t \geq r^{+}} \Delta(\mathrm{B} \alpha \leftrightarrow \bar{t})\right)
$$

where $r^{+}$is the successor of $r$. Now using axioms (B2)-(B4) and the fact that $\mathrm{B}(\varphi \otimes \varphi) \leftrightarrow \mathrm{B} \varphi \otimes \mathrm{B} \varphi$, and hence $\mathrm{B} \Delta \varphi \leftrightarrow \Delta \mathrm{B} \varphi$ is a theorem of $K 45\left(\mathrm{E}_{k}^{c}\right)$, the following expression can be derived from $\Delta(\mathrm{B} \alpha \leftrightarrow \bar{r})$.

$$
\mathrm{B}\left(\bigvee_{t \geq r} \Delta(\alpha \leftrightarrow \bar{t})\right) \wedge \neg \mathrm{B}\left(\bigvee_{t \geq r^{+}} \Delta(\alpha \leftrightarrow \bar{t})\right) .
$$

By the induction hypothesis, there exists for each $t \geq r$ a formula $\left(\alpha_{t}\right)^{\prime} \in \mathcal{L}_{\mathrm{O}}$ that is equivalent to $\Delta(\alpha \leftrightarrow \bar{t})$. Therefore, $\Delta(\mathrm{B} \alpha \leftrightarrow \bar{r})$ is equivalent to

$$
\mathrm{B}\left(\bigvee_{t \geq r}\left(\alpha_{t}\right)^{\prime}\right) \wedge \neg \mathrm{B}\left(\bigvee_{t \geq r^{+}}\left(\alpha_{t}\right)^{\prime}\right) \in \mathcal{L}_{O}^{\prime} .
$$

If, on the other hand $r=1$, then we have to show that $\Delta \mathrm{B} \alpha$ is equivalent to $\mathrm{B} \Delta \alpha$. As previously mentioned, this is a theorem of $K D 45\left(\leftarrow_{k}^{c}\right)$.

Finally, we show that the relationship between the "only knowing" operator O and Moore's stable expansions proved in [6] naturally extends to our framework. The next proposition shows that the belief set $\mathrm{Bel}_{S}$ for an epistemic state defined by a set of $€_{k}$-evaluations $S$ is indeed a stable fuzzy expansion of a premise $\varphi$ whenever $\Delta \varphi$ is all what is fully believed in the epistemic state $S$.

This proposition can easily be generalized to stable fuzzy expansions of sets of formulas.

Proposition 8. Suppose $A=\left\{\phi_{1}, \ldots, \phi_{n}\right\}$ is a set of formulas in $\mathcal{L}_{\mathrm{B}}$. Then Bel $\mathrm{B}_{S}$ is a stable fuzzy expansion of $A$ iff $\left\|\mathrm{O}\left(\Delta \phi_{1} \wedge \ldots \wedge \Delta \phi_{n}\right)\right\|_{S}=1$.

Proof. Since for a formula $\psi$ we have $\|\mathrm{O} \psi\|_{S}=\min \left(\|\mathrm{B} \psi\|_{S},\|\mathrm{~N} \neg \psi\|_{S}\right)$, we have the following chain of equivalences:

$$
\begin{aligned}
& \left\|\mathrm{O}\left(\Delta \phi_{1} \wedge \ldots \wedge \Delta \phi_{n}\right)\right\|_{S}=1 \\
& \Leftrightarrow\left\|\mathrm{B}\left(\Delta \phi_{1} \wedge \ldots \wedge \Delta \phi_{n}\right)\right\|_{S}=1 \text { and }\left\|\mathrm{N}\left(\neg\left(\Delta \phi_{1} \wedge \ldots \wedge \Delta \phi_{n}\right)\right)\right\|_{S}=1 \\
& \Leftrightarrow \forall v \in S:\left\|\Delta \phi_{1} \wedge \ldots \wedge \Delta \phi_{n}\right\|_{(v, S)}=1 \text { and } \\
& \quad \forall v \notin S:\left\|\Delta \phi_{1} \wedge \ldots \wedge \Delta \phi_{n}\right\|_{(v, S)}=0 \\
& \Leftrightarrow \forall v \in S, \forall i \in\{1, \ldots, n\}:\left\|\Delta \phi_{i}\right\|_{(v, S)}=1 \text { and }
\end{aligned}
$$




$$
\begin{array}{r}
\forall v \notin S, \exists j \in\{1, \ldots, n\}:\left\|\Delta \phi_{j}\right\|_{(v, S)}=0 \\
\Leftrightarrow \forall v \in S, \forall i \in\{1, \ldots, n\}:\left\|\phi_{i}\right\|_{(v, S)}=1 \text { and } \\
\forall v \notin S, \exists j \in\{1, \ldots, n\}:\left\|\phi_{j}\right\|_{(v, S)}<1
\end{array}
$$

Thus, assuming that $\left\|\mathrm{O}\left(\Delta \phi_{1} \wedge \ldots \wedge \Delta \phi_{n}\right)\right\|_{S}=1$, we can show that $S=\left\{v \in \Omega_{k} \mid\left\|\phi_{i}\right\|_{(v, S)}=1\right.$ for all $\left.i \in\{1, \ldots, n\}\right\}$. Indeed, by the previous statement we have $S \subseteq\left\{v \in \Omega_{k} \mid\left\|\phi_{i}\right\|_{(v, S)}=1\right.$ for all $\left.i \in\{1, \ldots, n\}\right\}$. If $w \in\left\{v \in \Omega_{k} \mid\right.$ $\left\|\phi_{i}\right\|_{(v, S)}=1$ for all $\left.i \in\{1, \ldots, n\}\right\}$ and suppose that $w \notin S$, then we would have that there exists a $j \in\{1, \ldots, n\}$ such that $\left\|\phi_{j}\right\|_{(w, S)}<1$, a contradiction. Hence $S=\left\{v \in \Omega_{k} \mid\left\|\phi_{i}\right\|_{(v, S)}=1\right.$ for all $\left.i \in\{1, \ldots, n\}\right\}$. Similar, one can also show that $S=\left\{v \in \Omega_{k} \mid\left\|\phi_{i}\right\|_{(v, S)}=1\right.$ for all $\left.i \in\{1, \ldots, n\}\right\}$ implies that for all $v \in S$ we have $\left\|\phi_{i}\right\|_{(v, S)}=1$ for all $i \in\{1, \ldots, n\}$ and for all $v \notin S$ we have that there exists a $j \in\{1, \ldots, n\}$ such that $\left\|\phi_{j}\right\|_{(v, S)}<1$. Hence

$$
\begin{aligned}
& \left\|\mathrm{O}\left(\Delta \phi_{1} \wedge \ldots \wedge \Delta \phi_{n}\right)\right\|_{S}=1 \\
& \quad \Leftrightarrow S=\left\{v \in \Omega_{k} \mid\left\|\phi_{i}\right\|_{(v, S)}=1 \text { for all } i \in\{1, \ldots, n\}\right\} \\
& \quad \Leftrightarrow S \text { is a fuzzy autoepistemic model of } A \\
& \quad \Leftrightarrow B e l_{S} \text { is a stable fuzzy expansion of } A,
\end{aligned}
$$

where the last equivalence follows from Proposition 4.

The next lemma proves interesting properties about graded beliefs the only knowing operator $\mathrm{O}$ captures inside the (monotonic) many-valued modal logic $\mathrm{O}\left(\mathfrak{E}_{k}^{c}\right)$ related to similar features of the (non-monotonic) fuzzy autoepistemic logic. We recall that a propositional formula $\varphi$ is $r$-satisfiable, for $r \in S_{k}$, if there exists an evaluation $w \in \Omega_{k}$ such that $w(\varphi)=r$.

Proposition 9. If $\varphi$ is a propositional formula and $r$-satisfiable for some $r \in S_{k}$, then $\mathrm{O}\left(E_{k}^{c}\right)$ proves the following formulas:

(i) $\mathrm{N}(\neg \Delta(\bar{r} \rightarrow \varphi)) \rightarrow(\mathrm{B} \varphi \rightarrow \bar{r})$

(ii) $\mathrm{O}(\Delta(\bar{r} \rightarrow \varphi)) \rightarrow(\mathrm{B} \varphi \leftrightarrow \bar{r})$

Proof. Let $\phi=\Delta(\bar{r} \leftrightarrow \varphi)$.

(i) Obviously, $\varphi$ is $r$-satisfiable iff $\phi$ is 1 -satisfiable, and since $\phi$ is Boolean, by axiom (v) of the logic $\mathrm{O}\left(\mathrm{E}_{k}^{c}\right)$ we have that $N(\neg \Delta(\bar{r} \leftrightarrow \varphi)) \rightarrow \neg \mathrm{B} \neg \Delta(\bar{r} \leftrightarrow \varphi)$ is a theorem. Since $\Delta(r \leftrightarrow \varphi) \rightarrow(\varphi \rightarrow \bar{r})$ is a theorem in $\mathfrak{七}_{k}^{c}$ we can derive that the following formula is a theorem in $\mathrm{O}\left(\mathrm{E}_{k}^{c}\right)$ :

$$
\neg \mathrm{B}(\neg \Delta(\bar{r} \leftrightarrow \varphi)) \rightarrow \neg \mathrm{B}(\neg(\varphi \rightarrow \bar{r}))
$$

Hence $\mathrm{O}\left(\mathrm{七}_{k}^{c}\right)$ proves

$$
\mathrm{N}(\neg \Delta(\bar{r} \leftrightarrow \varphi)) \rightarrow \neg \mathrm{B}(\neg(\varphi \rightarrow \bar{r})) .
$$

Since by axiom (B3),

$$
\neg \mathrm{B}(\neg(\varphi \rightarrow \bar{r})) \rightarrow(\mathrm{B} \varphi \rightarrow \bar{r})
$$

is a theorem, (i) is proved.

(ii) follows by observing that $\mathrm{O} \phi$ is equivalent to $\mathrm{B} \phi \wedge \mathrm{N} \neg \phi$, and hence $\mathrm{O} \phi \rightarrow \mathrm{B} \phi$ is a theorem, i.e. $\mathrm{B} \Delta(r \leftrightarrow \varphi)$. Since $\Delta(\bar{r} \leftrightarrow \varphi) \rightarrow(\bar{r} \rightarrow \varphi)$ is a theorem in $\mathrm{Ł}_{k}^{c}$ we obtain that

$$
\mathrm{B}(\Delta(\bar{r} \leftrightarrow \varphi)) \rightarrow \mathrm{B}(\bar{r} \rightarrow \varphi)
$$

is a theorem in $\mathrm{O}\left(\mathrm{E}_{k}^{c}\right)$. Since $\mathrm{B}(\bar{r} \rightarrow \varphi)$ is equivalent to $\bar{r} \rightarrow \mathrm{B} \varphi$ by axiom (B3), we obtain that

$$
\mathrm{B}(\Delta(r \leftrightarrow \varphi)) \rightarrow(\bar{r} \rightarrow \mathrm{B} \varphi)
$$

is a theorem in $\mathrm{O}\left(\mathfrak{E}_{k}^{c}\right)$. Finally, taking into account (i), we get that $\mathrm{O}\left(\mathfrak{E}_{k}^{c}\right)$ proves $\mathrm{O}(\Delta(\bar{r} \rightarrow \varphi)) \rightarrow(\mathrm{B} \varphi \leftrightarrow \bar{r})$. 
Example 5. We now present an alternative encoding for the scenario in Example 4 using the "only knowing" logic. If we let

$$
\begin{aligned}
\phi_{A}= & \Delta\left(\mathrm{B} \neg e_{2}\right) \wedge \Delta\left(\neg \mathrm{B} e_{1} \rightarrow \neg e_{1}\right) \wedge \Delta\left(\neg \mathrm{B} e_{2} \rightarrow \neg e_{2}\right) \wedge \Delta\left(\neg \mathrm{B} e_{3} \rightarrow \neg e_{3}\right) \\
& \wedge \Delta\left(\overline{0.7} \rightarrow\left(e_{1} \vee e_{2}\right)\right) \wedge \Delta\left(\overline{0.4} \rightarrow\left(e_{2} \vee e_{3}\right)\right) \wedge \Delta\left(\overline{0.3} \rightarrow\left(e_{1} \vee e_{3}\right)\right)
\end{aligned}
$$

Then, by the above proposition, if we "only know" $\phi_{A}$, i.e. if we assume $\mathrm{O} \phi_{A}$, one can infer (both semantically and syntactically) for example the following graded beliefs:

$$
\overline{0.7} \leftrightarrow \mathrm{B} e_{1}, \neg \mathrm{B} e_{2}, \overline{0.4} \leftrightarrow \mathrm{B} e_{3}, \overline{0.3} \leftrightarrow \mathrm{B} \neg e_{1}, \mathrm{~B} \neg e_{2} \text {, and } \overline{0.6} \leftrightarrow \mathrm{B} \neg e_{3} .
$$

As an example, let us now use Proposition 8 to find the stable fuzzy expansion of

$$
A=\left\{\neg \mathrm{B} e_{1} \rightarrow \neg e_{1}, \neg \mathrm{B} e_{2} \rightarrow \neg e_{2}, \neg \mathrm{B} e_{3} \rightarrow \neg e_{3}, \mathrm{~B} \neg e_{2}, \overline{0.7} \rightarrow e_{1} \vee e_{2}, \overline{0.4} \rightarrow e_{2} \vee e_{3}, \overline{0.3} \rightarrow e_{1} \vee e_{3}\right\}
$$

from Example 4.

Suppose $S$ is a fuzzy autoepistemic model of $A$, then by Proposition 8 it should hold that $\left\|\mathrm{O} \phi_{A}\right\|_{S}=1$. By the semantics of the operator $\mathrm{O}$, it follows that

$$
\begin{aligned}
& \| \mathrm{B}\left(\Delta\left(\neg \mathrm{B} e_{1} \rightarrow \neg e_{1}\right) \wedge \Delta\left(\neg \mathrm{B} e_{2} \rightarrow \neg e_{2}\right) \wedge \Delta\left(\neg \mathrm{B} e_{3} \rightarrow \neg e_{3}\right) \wedge \Delta\left(\mathrm{B} \neg e_{2}\right)\right. \\
& \left.\quad \wedge \Delta\left(\overline{0.7} \rightarrow\left(e_{1} \vee e_{2}\right)\right) \wedge \Delta\left(\overline{0.4} \rightarrow\left(e_{2} \vee e_{3}\right)\right) \wedge \Delta\left(\overline{0.3} \rightarrow\left(e_{1} \vee e_{3}\right)\right)\right) \|_{S}=1
\end{aligned}
$$

and hence by the semantics of the operator B we obtain for all $v \in S$ and for all $\phi \in A$ that $\|\Delta \phi\|_{(v, S)}=1$ and hence that $\|\phi\|_{(v, S)}=1$. The same computations as in Example 4 imply that this is only possible if

$$
S=\left\{v \in \Omega_{k} \mid v\left(e_{1}\right)=0.7, v\left(e_{2}\right)=0, v\left(e_{3}\right)=0.4\right\} .
$$

To obtain that $S$ is a fuzzy autoepistemic model of $A$, it remains to be shown that

$$
\begin{aligned}
\| \mathrm{N} & \neg\left(\Delta\left(\neg \mathrm{B} e_{1} \rightarrow \neg e_{1}\right) \wedge \Delta\left(\neg \mathrm{B} e_{2} \rightarrow \neg e_{2}\right) \wedge \Delta\left(\neg \mathrm{B} e_{3} \rightarrow \neg e_{3}\right) \wedge \Delta\left(\mathrm{B} \neg e_{2}\right)\right. \\
\quad & \left.\wedge \Delta\left(\overline{0.7} \rightarrow\left(e_{1} \vee e_{2}\right)\right) \wedge \Delta\left(\overline{0.4} \rightarrow\left(e_{2} \vee e_{3}\right)\right) \wedge \Delta\left(\overline{0.3} \rightarrow\left(e_{1} \vee e_{3}\right)\right)\right) \|_{S}=1
\end{aligned}
$$

Or in other words, it has to be shown that for all $w \notin S$ there exists $\phi \in A$ such that $\|\phi\|_{(w, S)}<1$. Suppose this is not the case and there exists $w \notin S$ such that for all $\phi \in A$ it holds that $\|\phi\|_{(w, S)}=1$. Since for all $i \in\{1,2,3\}$ we have $\left\|\neg \mathrm{B} e_{i} \rightarrow \neg e_{i}\right\|_{(w, S)}=1$ it follows that $w\left(e_{i}\right) \leq \inf _{z \in S} z\left(e_{i}\right)=v\left(e_{i}\right)$. Since $w \neq v$ there exists $j \in\{1,3\}$ such that $w\left(e_{j}\right)<v\left(e_{j}\right)$ and $w\left(e_{2}\right)=0$. If $j=1$, since $\left\|\overline{0.7} \rightarrow e_{1} \vee e_{2}\right\|_{w, S}=1$, we obtain

$$
w\left(e_{1}\right)<v\left(e_{1}\right)=0.7 \leq w\left(e_{1} \vee e_{2}\right)=w\left(e_{1}\right),
$$

a contradiction. For $j=3$ we obtain a similar contradiction.

\section{Related work}

Many-valued modal logics have appeared in the literature under different forms and contexts. In [25,26], a modal logic with truth values in finite Heyting algebras is introduced. These modal systems are then used for dealing with opinions of experts with a dominance relation among them. Other papers mainly offer theoretical frameworks. For instance, in the last years there has been some work on fuzzy modal logics with generalized Kripke semantics, see e.g. [17]. In particular, in [17], they systematically investigate many-valued modal logics over a residuated lattice, dealing with accessibility relations that can take values in this lattice as well as relations taking only values $T$ and $\perp$ in the lattice. In the current paper we have focused on modal systems based on a finite set of linearly ordered truth values with Łukasiewicz logic semantics for connectives which generate the class of finite MV-algebras [21]. These systems represent a good compromise between expressive power and nice logical properties. The infinitely-valued case offers some problems, see e.g. [27] where infinitely-valued Łukasiewicz logic is extended with a modality and the results involve an infinitary deduction rule. Another example is the case of S5 with total accessibility relations [28]. In that paper the fact that formulas are in correspondence with formulas of the monadic fuzzy predicate calculus is used to propose a sound and complete axiomatization by interpreting the modality as " $\forall$ ". However, for KD45 and K45 this trick does not work since in these cases the modality cannot be interpreted in such a way. On the other hand, a closely 
related work is Maruyama's paper [29], where modal logics for belief based on a finitely-valued Heyting algebra of truth values which means that the truth values are not necessarily linearly ordered, are considered. The formalization is very similar, but he also deals with common belief. Here we rather focus on providing a formal basis for the fuzzy generalization of autoepistemic logic developed in [9].

Autoepistemic logic is among the most extensively studied nonmonotonic formal systems and is known to be equivalent to several other modal nonmonotonic reasoning systems such as the logic of only knowing [6] and is closely related to default logic [30]. Besides in [9], many-valued extensions of autoepistemic logic have also been addressed by e.g. Fitting [31] in the context of finite Heyting algebras of truth values. The latter is actually an extension of the earlier mentioned work in $[25,26]$ to the nonmonotonic setting. Reflexive autoepistemic logic has almost identical semantic characterizations of stable expansions as autoepistemic logic but defines the modality so that it models knowledge rather than belief. It can also be used to characterize the semantics of ASP [32] and has been generalized in [33] using finite Heyting algebras.

\section{Concluding remarks}

In this paper we have introduced Hilbert-style axiomatizations for fuzzy modal logics of belief as well as for a "logic of only knowing" based on finitely-valued Łukasiewicz logic. In particular, we have introduced generalizations of the main classical propositional modal logics of belief (K45, KD45, S5) in order to model the notion of belief on fuzzy propositions, in the sense of admitting partial degrees of truth between 0 (fully false) and 1 (fully true). We have shown that their Kripke style semantics can also be used to provide a possible world semantics for fuzzy autoepistemic logic, in line with the original work of Moore [4]. We have also developed a fuzzy version of Levesque's (propositional) logic of "only knowing", proving soundness and completeness, and in particular we have generalized bridges with autoepistemic logic established in [6] for the classical case by characterizing fuzzy stable expansions in terms of models of suitable "only knowing" formulas.

The paper also leaves some interesting open problems to be dealt with in future developments. A first question is considering whether fuzzy accessibility relations instead of crisp ones would make the approach significantly more general. From an application point of view, keeping accessibility relations crisp makes it easier to interpret the truth degree of a formula. If we would have fuzzy accessibility relations in addition to graded truth, the truth degree of a formula $\mathrm{B} \phi$ depends on the degree to which $\phi$ is believed and on how certain we are of that belief. Also, note that crisp accessibility relations seem to be sufficient for practical purposes, and in particular for making the link with fuzzy autoepistemic logic in the sense of [9] and fuzzy answer set programming in the sense of [10]. On the other hand, it also seems interesting to consider fuzzy accessibility relations e.g. under the form of a possibility distribution on the set of worlds, since this would allow us to consider scenarios in which beliefs can be both graded and uncertain. Another type of generalization that may be interesting to look at would be to start from other logics than finitely-valued Łukasiewicz logic. Although, if one wants to remain in a finitely-valued framework, $(k+1)$-valued Łukasiewicz logic seems to be the best choice regarding the expressive power, unless one prefers a purely ordinal level. In this case one could use Gödel caculi and related logics, possibly equipped with an extra involutive negation. Moving to infinitely-valued logics, in particular to the [0,1]-valued Łukasiewicz logic $€$, may bring technical difficulties as $€$ is not strongly complete for arbitrary sets of premises, an important property in our current approach.

\section{Acknowledgements}

The authors are grateful to anonymous reviewers for their helpful comments and suggestions that have helped to improve the final presentation of this paper.

\section{Appendix A. Proofs of some results}

Lemma 1. Let $L$ be any of the logics $K 45\left(E_{k}^{c}\right), K D 45\left(E_{k}^{c}\right)$, $S 5\left(E_{k}^{c}\right)$. Suppose $T \cup\{\psi\}$ is a set of formulas from $\mathcal{L}_{\mathrm{B}}$ and let $\Lambda_{L}=\left\{\phi^{*} \mid \vdash_{L} \phi\right\}$. Then it holds that $T \vdash_{L} \psi$ iff $T^{*} \cup \Lambda_{L} \vdash \psi^{*}$. 
Proof. Suppose a proof for $\psi$ in $L$ from $T$ has the form $\Gamma=\left(\gamma_{1}, \ldots, \gamma_{m}\right)$. A proof for $\psi^{*}$ in $£_{k}^{c}$ from $T^{*} \cup \Lambda_{L}$ is then easily obtained by replacing all formulas $\gamma_{i}$ in $\Gamma$ by $\gamma_{i}^{*}$. Indeed, notice that for all $i$ it holds that $\gamma_{i}$ is one of the following

- an element in $T$, and then $\gamma_{i}^{*}$ is an element of $T^{*}$;

- an instance of an axiom of $L$, and then $\gamma_{i}^{*}$ is an element of $\Lambda_{L}$;

- a formula obtained by modus ponens from $\gamma_{j}=\gamma_{k} \rightarrow \gamma_{i}$ and $\gamma_{k}$, with $j, k<i$. Then $\gamma_{i}^{*}$ is also obtained by modus ponens from $\gamma_{j}^{*}$ and $\gamma_{k}^{*}$;

- a formula $\mathrm{B} \gamma_{j}$ obtained by necessitation from a theorem $\gamma_{j}(j<i)$ of $\mathrm{L}$. Then $\gamma_{i}$ is a theorem in $L$ and $\gamma_{i}^{*}$ is an element of $\Lambda_{L}$.

Conversely, suppose there is a proof $\Phi=\left(\phi_{1}, \ldots, \phi_{n}\right)$ for $\psi^{*}$ in $Ł_{k}^{c}$ from $T^{*} \cup \Lambda_{L}$. The sequence $\Phi$ can then be converted to a proof for $\psi$ in $L$ from $T$ as follows.

- If $\phi_{i} \in \Lambda_{L}$, i.e. $\phi_{i}=\gamma_{i}^{*}$ with $\vdash_{L} \gamma_{i}$, then replace $\phi_{i}$ by $\gamma_{i}$ and add a proof for $\gamma_{i}$.

- Otherwise, replace $\phi_{i}=\gamma_{i}^{*}$ by $\gamma_{i}$.

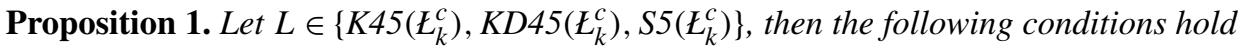

1. If $L$ contains axiom $(T)$ then $R_{\text {can }}^{L}$ is reflexive.

2. If $L$ contains axiom (4) then $R_{\text {can }}^{L}$ is transitive.

3. If $L$ contains axiom (5) then $R_{\text {can }}^{L}$ is Euclidean.

4. If $L$ contains axiom $(D)$ then $R_{\text {can }}^{L}$ is serial.

Proof. In this proof we frequently use the result from Lemma 2: for every $v \in W_{\text {can }}^{L}$ and every formula $\phi$ it holds that $v\left(\phi^{*}\right)=\|\phi\|_{M_{\text {can }}^{L}, v}$.

1. Let $w \in W_{\text {can }}^{L}$. Suppose that $w\left((\mathrm{~B} \phi)^{*}\right)=1$, we show $w\left(\phi^{*}\right)=1$. Then it follows by the construction of the canonical model that $R_{c a n}^{L}(w, w)=1$. Since $L$ contains axiom $(\mathrm{T})$, we have $(\mathrm{B} \phi \rightarrow \phi)^{*} \in \Lambda_{L}$ and it follows that $1=$ $w\left((\mathrm{~B} \phi \rightarrow \phi)^{*}\right)=\|\mathrm{B} \phi \rightarrow \phi\|_{M_{c a n}^{L}, w}$. Hence $1=w\left((\mathrm{~B} \phi)^{*}\right)=\|\mathrm{B} \phi\|_{M_{c a n}^{L}, w} \leq\|\phi\|_{M_{c a n}^{L}, w}^{L}=w\left(\phi^{*}\right)$.

2. Let $w_{1}, w_{2}, w_{3} \in W_{\text {can }}^{L}$ such that $R_{\text {can }}^{L}\left(w_{1}, w_{2}\right)=R_{\text {can }}^{L}\left(w_{2}, w_{3}\right)=1$. We show that $R_{\text {can }}^{L}\left(w_{1}, w_{3}\right)=1$. Suppose that $w_{1}\left((\mathrm{~B} \phi)^{*}\right)=1$, we show $w_{3}\left(\phi^{*}\right)=1$. Since $L$ contains axiom (4), we have $(\mathrm{B} \phi \rightarrow \mathrm{BB} \phi)^{*} \in \Lambda_{L}$ and it follows that $1=w_{1}\left((\mathrm{~B} \phi \rightarrow \mathrm{BB} \phi)^{*}\right)=\|\mathrm{B} \phi \rightarrow \mathrm{BB} \phi\|_{M_{c a n}^{L}, w_{1}}$, and hence that $1=w_{1}\left((\mathrm{~B} \phi)^{*}\right)=\|\mathrm{B} \phi\|_{M_{c a n}^{L}, w_{1}} \leq\|\mathrm{BB} \phi\|_{M_{c a n}^{L}, w_{1}}=$ $w_{1}\left((\mathrm{BB} \phi)^{*}\right)$. Since $R_{\text {can }}^{L}\left(w_{1}, w_{2}\right)=1$, we then have that $w_{2}\left((\mathrm{~B} \phi)^{*}\right)=1$ and subsequently, since $R_{\text {can }}^{L}\left(w_{2}, w_{3}\right)=1$, that $w_{3}\left(\phi^{*}\right)=1$.

3. Let $w_{1}, w_{2}, w_{3} \in W_{\text {can }}^{L}$ such that $R_{\text {can }}^{L}\left(w_{1}, w_{2}\right)=R_{\text {can }}^{L}\left(w_{1}, w_{3}\right)=1$. We show that $R_{\text {can }}^{L}\left(w_{2}, w_{3}\right)=1$. Suppose that $w_{2}\left((\mathrm{~B} \phi)^{*}\right)=1$, we show $w_{3}\left(\phi^{*}\right)=1$. By definition of $\mathrm{B}$,

$$
\|\mathrm{B} \neg \mathrm{B} \phi\|_{M_{c a n}^{L}, w_{1}}=\inf \left\{\|\neg \mathrm{B} \phi\|_{M_{c a n}^{L}, w} \mid R_{c a n}^{L}\left(w_{1}, w\right)=1\right\},
$$

hence in particular $\|\mathrm{B} \neg \mathrm{B} \phi\|_{M_{c a n}^{L}, w_{1}} \leq\|\neg \mathrm{B} \phi\|_{M_{c a n}^{L}, w_{2}}$. Now since $\|\neg \mathrm{B} \phi\|_{M_{c a n}^{L}, w_{2}}=1-\|\mathrm{B} \phi\|_{M_{c a n}^{L}, w_{2}}=1-$ $w_{2}\left((\mathrm{~B} \phi)^{*}\right)=0$, we obtain $\|\mathrm{B} \neg \mathrm{B} \phi\|_{M_{c a n}^{L}, w_{1}}^{L}=0$. But since $(\neg \mathrm{B} \neg \mathrm{B} \phi \rightarrow \mathrm{B} \phi)^{*} \in \Lambda_{L}$ because of axiom (5), it follows that

$$
1=w_{1}\left((\neg \mathrm{B} \neg \mathrm{B} \phi \rightarrow \mathrm{B} \phi)^{*}\right)=\|\neg \mathrm{B} \neg \mathrm{B} \phi \rightarrow \mathrm{B} \phi\|_{M_{c a n}^{L}, w_{1}}
$$

and hence $1=\|\neg \mathrm{B} \neg \mathrm{B} \phi\|_{M_{c a n}^{L}, w_{1}} \leq\|\mathrm{B} \phi\|_{M_{c a n}^{L}, w_{1}}=w_{1}\left((\mathrm{~B} \phi)^{*}\right)$. Finally, since $R_{c a n}^{L}\left(w_{1}, w_{3}\right)=1$, it then follows that $w_{3}\left(\phi^{*}\right)=1$.

4. Let $w_{1} \in W_{\text {can }}^{L}$. We show that there exists $w_{2} \in W_{\text {can }}^{L}$ such that $R_{\text {can }}^{L}\left(w_{1}, w_{2}\right)=1$. Since by axiom (D) we have $(\neg \mathrm{B} \neg \overline{1})^{*} \in \Lambda_{L}$, it follows that $1=w_{1}\left((\neg \mathrm{B} \neg \overline{1})^{*}\right)=\|\neg \mathrm{B} \neg \overline{1}\|_{M_{c a n}^{L}, w_{1}}$, and thus $0=\|\mathrm{B} \neg \overline{1}\|_{M_{c a n}^{L}, w_{1}}=\inf \left\{\|\overline{0}\|_{M_{c a n}^{L}, w} \mid\right.$ $\left.R_{c a n}^{L}\left(w_{1}, w\right)=1\right\}$. Therefore the latter set must be non-empty, and hence there must exist $w_{2} \in W_{\text {can }}^{L}$ such that $R_{\text {can }}^{L}\left(w_{1}, w_{2}\right)=1$. 
Proposition 2. $K 45\left({E_{k}^{c}}^{c}\right), K D 45\left(\leftarrow_{k}^{c}\right)$ and $S 5\left(E_{k}^{c}\right)$ are sound and complete w.r.t. the classes $\mathbb{M}_{\mathrm{et}}^{s}$, $\mathbb{M}_{\mathrm{est}}^{s}$ and $\mathbb{M}_{\mathrm{rsyt}}^{s}$ respectively.

Proof. We only prove the case of $K D 45\left(\mathrm{E}_{k}^{c}\right)$, the other cases being easy variations. By Theorem 2, it is sufficient to show that $\mathbb{M}_{\text {est }}$ and $\mathbb{M}_{\text {est }}^{s}$ have the same tautologies. Since $\mathbb{M}_{\text {est }}^{s}$ is a subclass of $\mathbb{M}_{\text {est }}$, we only have to show that if for a formula $\phi$ there exists an $M=(W, e, R) \in \mathbb{M}_{\text {est }}$ and $w \in W$ such that $\|\phi\|_{M, w}<1$, then there exist an $M^{\prime}=\left(W^{\prime}, e^{\prime}, R^{\prime}\right) \in \mathbb{M}_{\text {est }}^{s}$ and $w^{\prime} \in W^{\prime}$ such that $\|\phi\|_{M^{\prime}, w^{\prime}}<1$.

Suppose such a Kripke model $M=(W, e, R) \in \mathbb{M}_{\text {est }}$ and $w \in W$ are given. Define $E=\{v \in W \mid R(w, v)=1\}$. By the seriality of $R$ we have $E \neq \emptyset$. We define $M^{\prime}$ as follows: $W^{\prime}=\{w\} \cup E, e^{\prime}: W^{\prime} \times V \rightarrow S_{k}:(w, p) \mapsto e(w, p)$ and $R^{\prime}=W^{\prime} \times E$.

Notice that for any $v \in E$ we have $E=\{z \in W \mid R(v, z)=1\}$. Indeed, for every $z \in E$ we have $R(w, z)=1$ and since $R$ is Euclidean and $R(w, v)=1$ (because $v \in E$ ) it follows that $R(v, z)=1$ as well, and hence $E \subseteq\{z \in W$ $R(v, z)=1\}$. On the other hand, since $R$ is transitive, if $R(v, z)=1$, then since $R(w, v)=1$ it follows $R(w, z)=1$ and thus $z \in E$, and hence $\{z \in W \mid R(z, v)=1\} \subseteq E$. Therefore, $E=\{z \in W \mid R(v, z)=1\}$ for all $v \in E$.

We will now use this result to show by structural induction that for each $\psi \in \mathcal{L}_{B}$ it holds that $\|\psi\|_{M, v}=\|\psi\|_{M^{\prime}, v}$ for every $v \in E$. The only notable case is when $\psi=\mathrm{B} \alpha$, but this follows by the fact that $E=\{z \in W \mid R(v, z)=1\}$ for all $v \in E$ and by the hypothesis:

$$
\begin{aligned}
\|\mathrm{B} \alpha\|_{M, v} & =\inf \left\{\|\alpha\|_{M, z} \mid R(v, z)=1\right\} \\
& =\inf \left\{\|\alpha\|_{M, z} \mid z \in E\right\} \\
& =\inf \left\{\|\alpha\|_{M^{\prime}, z} \mid z \in E\right\} \\
& =\inf \left\{\|\alpha\|_{M^{\prime}, z} \mid R^{\prime}(v, z)=1\right\} \\
& =\|\operatorname{B} \alpha\|_{M^{\prime}, v}
\end{aligned}
$$

where we use that $R^{\prime}=W^{\prime} \times E$.

We will use this last result to show that $\|\psi\|_{M, w}=\|\psi\|_{M^{\prime}, w}$ for all $\psi \in \mathcal{L}_{B}$. In particular, it then follows that $\|\phi\|_{M^{\prime}, w}=\|\phi\|_{M, w}<1$. We will show this by structural induction. Again, the only notable case is when $\psi=\mathrm{B} \alpha$ :

$$
\begin{aligned}
\|\mathrm{B} \alpha\|_{M^{\prime}, w} & =\inf \left\{\|\alpha\|_{M^{\prime}, z} \mid R^{\prime}(w, z)=1\right\} \\
& =\inf \left\{\|\alpha\|_{M^{\prime}, z} \mid z \in E\right\} \\
& =\inf \left\{\|\alpha\|_{M, z} \mid z \in E\right\} \\
& =\inf \left\{\|\alpha\|_{M, z} \mid R(w, z)=1\right\} \\
& =\|\operatorname{B} \alpha\|_{M, w}
\end{aligned}
$$

Lemma 2. For any formula $\phi \in \mathcal{L}_{\mathrm{B}}$ and truth value $r \in S_{k}$, there exists a formula $\left(\phi_{r}\right)^{\prime} \in \mathcal{L}_{B}^{\prime}$ such that for each Kripke model $M=(W, e, R) \in \mathbb{M}$ and each world $w \in W$ it holds that

$$
\left\|\phi_{r}\right\|_{M, w}=\left\|\left(\phi_{r}\right)^{\prime}\right\|_{M, w} .
$$

Proof. We show this lemma by induction on the structure of the formula.

- If $\phi=p \in V$, then we can choose $\left(\phi_{r}\right)^{\prime}=\Delta(p \leftrightarrow \bar{r})$.

- Suppose that $\phi=\bar{c}$ with $c \in S_{k}$. Then $\left\|\phi_{r}\right\|_{M, w}=1$ iff $r=c$. If $c=r$, then we can choose any tautology in $\mathcal{L}_{B}^{\prime}$, e.g. $\left(\phi_{r}\right)^{\prime}=\Delta(p \leftrightarrow \overline{1}) \leftrightarrow \Delta(p \leftrightarrow \overline{1})$ with $p \in V$ arbitrary. If $c \neq r$, then we can choose any contradiction, e.g. $\left(\phi_{r}\right)^{\prime}=\neg(\Delta(p \leftrightarrow \overline{1}) \leftrightarrow \Delta(p \leftrightarrow \overline{1}))$ with $p \in V$ arbitrary.

- Suppose the claim holds for formulas $\phi$ and $\psi$, i.e. for every $s \in S_{k}$ there exist some $\left(\phi_{s}\right)^{\prime}$ and $\left(\psi_{s}\right)^{\prime}$ in $\mathcal{L}_{B}^{\prime}$ such that $\left\|\phi_{S}\right\|_{M, w}=\left\|\left(\phi_{s}\right)^{\prime}\right\|_{M, w}$ and $\left\|\psi_{s}\right\|_{M, w}=\left\|\left(\psi_{s}\right)^{\prime}\right\|_{M, w}$. We show that the lemma also holds for $\alpha=\phi \rightarrow \psi$. Indeed, for $r \in S_{k}$ we choose

$$
\left(\alpha_{r}\right)^{\prime}=\bigvee_{s, t \in S_{k}}\left\{\left(\phi_{s}\right)^{\prime} \wedge\left(\psi_{t}\right)^{\prime} \mid r=(s \Rightarrow t)\right\}
$$


Then we have

$$
\begin{aligned}
\left\|\alpha_{r}\right\|_{M, w}=1 & \Leftrightarrow\left(\|\phi\|_{M, w} \Rightarrow\|\psi\|_{M, w}\right)=r \\
& \Leftrightarrow \exists s, t \in S_{k}:\|\phi\|_{M, w}=s,\|\psi\|_{M, w}=t, r=(s \Rightarrow t) \\
& \Leftrightarrow \exists s, t \in S_{k}:\left\|\phi_{s}\right\|_{M, w}=1,\left\|\psi_{t}\right\|_{M, w}=1, r=(s \Rightarrow t) \\
& \Leftrightarrow \exists s, t \in S_{k}:\left\|\left(\phi_{s}\right)^{\prime}\right\|_{M, w}=1,\left\|\left(\psi_{t}\right)^{\prime}\right\|_{M, w}=1, r=(s \Rightarrow t) \\
& \Leftrightarrow\left\|\left(\alpha_{r}\right)^{\prime}\right\|_{M, w}=1
\end{aligned}
$$

Since $\alpha_{r}$ and $\left(\alpha_{r}\right)^{\prime}$ are both Boolean formulas it follows that $\left\|\alpha_{r}\right\|_{M, w}=\left\|\left(\alpha_{r}\right)^{\prime}\right\|_{M, w}$.

- Suppose the claim holds for a formula $\phi$, i.e. for every $s \in S_{k}$ there exist some $\left(\phi_{s}\right)^{\prime}$ in $\mathcal{L}_{B}^{\prime}$ such that $\left\|\phi_{s}\right\|_{M, w}=$ $\left\|\left(\phi_{s}\right)^{\prime}\right\|_{M, w}$. We show that it also holds for $\alpha=\mathrm{B} \phi$. Suppose that $r \in S_{k}$. If $r=1$, then consider $\left(\alpha_{1}\right)^{\prime}=\mathrm{B}\left(\phi_{1}\right)^{\prime} \in$ $\mathcal{L}_{B}^{\prime}$ :

$$
\begin{aligned}
\left\|\alpha_{1}\right\|_{M, w}=1 & \Leftrightarrow\|\mathrm{B} \phi\|_{M, w}=1 \\
& \Leftrightarrow \inf \left\{\|\phi\|_{M, v} \mid R(w, v)=1\right\}=1 \\
& \Leftrightarrow \forall v \in W \text { such that } R(w, v)=1:\|\phi\|_{M, v}=1 \\
& \Leftrightarrow \forall v \in W \text { such that } R(w, v)=1:\left\|\phi_{1}\right\|_{M, v}=1 \\
& \Leftrightarrow \forall v \in W \text { such that } R(w, v)=1:\left\|\left(\phi_{1}\right)^{\prime}\right\|_{M, v}=1 \\
& \Leftrightarrow \inf \left\{\left\|\left(\phi_{1}\right)^{\prime}\right\|_{M, v} \mid R(w, v)=1\right\}=1 \\
& \Leftrightarrow\left\|\mathrm{B}\left(\phi_{1}\right)^{\prime}\right\|_{M, w}=1 \\
& \Leftrightarrow\left\|\left(\alpha_{r}\right)^{\prime}\right\|_{M, w}=1
\end{aligned}
$$

If $r<1$, then let $r^{+}$be the successor of $r$, i.e. if $r=\frac{i}{k}$, then $r^{+}=\frac{i+1}{k}$. Define formulas $\phi_{\geq r}, \phi_{>r}, \phi_{\leq r}$ and $\phi_{<r}$ as disjunctions ${ }^{8}$ of formulas $\phi_{s}$, e.g. $\phi_{\geq r}=\bigvee_{s \geq r} \phi_{s}$ and $\left\|\phi_{\geq r}\right\|_{M, w}=1$ iff $\|\phi\|_{M, w} \geq r$. Then we have that $\alpha_{r}$ is equivalent to $\alpha_{\geq r} \wedge \neg\left(\alpha_{\geq r^{+}}\right)$.

First note that for each $s \in S_{k}$ and each formula $\psi$ we have

$$
\begin{aligned}
\left\|(\mathrm{B} \psi)_{\geq s}\right\|_{M, w}=1 & \Leftrightarrow\|\mathrm{B} \psi\|_{M, w} \geq s \\
& \Leftrightarrow \inf \left\{\|\psi\|_{M, v} \mid R(w, v)=1\right\} \geq s \\
& \Leftrightarrow \forall v \in W \text { such that } R(w, v)=1:\|\psi\|_{M, v} \geq s \\
& \Leftrightarrow \forall v \in W \text { such that } R(w, v)=1:\left\|\psi_{\geq s}\right\|_{M, v}=1 \\
& \Leftrightarrow \forall v \in W \text { such that } R(w, v)=1:\left\|\bigvee_{t \geq s} \psi_{t}\right\|_{M, v}=1 \\
& \Leftrightarrow\left\|\mathrm{B}\left(\bigvee_{t \geq s} \psi_{t}\right)\right\|_{M, w}=1
\end{aligned}
$$

Hence we obtain

$$
\begin{aligned}
\left\|\alpha_{r}\right\|_{M, w}=1 & \Leftrightarrow\left\|(\mathrm{B} \phi)_{\geq r}\right\|_{M, w}=1 \text { and }\left\|\neg\left((\mathrm{B} \phi)_{\geq r^{+}}\right)\right\|_{M, w}=1 \\
& \Leftrightarrow\left\|\mathrm{B}\left(\bigvee_{t \geq r} \phi_{t}\right)\right\|_{M, w}=1 \text { and }\left\|\neg\left(\mathrm{B}\left(\bigvee_{t \geq r^{+}} \phi_{t}\right)\right)\right\|=1 \\
& \Leftrightarrow\left\|\mathrm{B}\left(\bigvee_{t \geq r} \phi_{t}\right) \wedge \neg \mathrm{B}\left(\bigvee_{t \geq r^{+}} \phi_{t}\right)\right\|_{M, w}=1
\end{aligned}
$$

By the induction hypothesis it follows that there exists $\left(\alpha_{r}\right)^{\prime} \in \mathcal{L}_{B}^{\prime}$ such that $\left\|\alpha_{r}\right\|_{M, w}=\left\|\left(\alpha_{r}\right)^{\prime}\right\|_{M, w}$.

8 Note that for Boolean formulas, $\oplus, \vee$ and the classical disjunction coincide. The same result holds for $\otimes, \wedge$ and the classical conjunction. 


\section{References}

[1] R. Moore, Semantical considerations on nonmonotonic logic, in: Proceedings of the Eight International Joint Conference on Artificial Intelligence, 1983, pp. 272-279.

[2] K. Konolige, Autoepistemic logic, in: Gabbay, et al. (Eds.), Handbook of Logic in Artificial Intelligence and Logic Programming, vol. 3, Oxford Science Publications, 1994, pp. 217-418.

[3] G. Shvarts, Autoepistemic modal logics, in: Proceedings of Theoretical Aspects of Reasoning About Knowledge, 1990, pp. 97-109.

[4] R. Moore, Possible-world semantics in autoepistemic logic, in: Proceedings of the Non-Monotonic Reasoning Workshop, 1984 , pp. 344-354.

[5] J. Halpern, Y. Moses, Towards a theory of knowledge and ignorance: preliminary report, in: Proceedings of the International Workshop on Nonmonotonic Reasoning, 1984, pp. 125-143.

[6] H. Levesque, All I know: a study in autoepistemic logic, Artif. Intell. (1990) 263-309.

[7] C. Baral, M. Gelfond, Logic programming and knowledge representation, J. Log. Program. 19 (1994) 73-148.

[8] M. Gelfond, V. Lifschitz, The stable model semantics for logic programming, in: Proceedings of the Fifth International Conference and Symposium on Logic Programming, 1988, pp. 1070-1080.

[9] M. Blondeel, S. Schockaert, M. De Cock, D. Vermeir, Fuzzy autoepistemic logic and its relation to fuzzy answer set programming, Fuzzy Sets Syst. 239 (2014) 51-80.

[10] D. Van Nieuwenborgh, M. De Cock, D. Vermeir, An introduction to fuzzy answer set programming, Ann. Math. Artif. Intell. 50 (3-4) (2007) 363-388.

[11] P. Hájek, Metamathematics of Fuzzy Logic, Trends in Logic, 1998.

[12] R. Cignoli, I. D’Ottaviano, D. Mundici, Algebraic Foundations of Many-Valued Reasoning, Kluwer, Dordrecht, 2000.

[13] R. Grigolia, Algebraic analysis of Łukasiewicz-Tarski n-valued logical systems, in: R. Wòjcicki, G. Malinowski (Eds.), Selected Papers on Łukasiewicz Sentencial Calculi, Polish Academy of Science, Osslineum, Wrocław, 1977, pp. 81-91.

[14] T. Flaminio, Strong non-standard completeness for fuzzy logics, Soft Comput. 12 (2008) 321-333.

[15] A. Monteiro, Algèbres de Heyting symétriques, Port. Math. 39 (1-4) (1980) 1-239.

[16] M. Baaz, Infinite-valued Gödel logic with 0-1-projections and relativisations, in: P. Hájek (Ed.), Gödel '96: Logical Foundations of Mathematics, Computer Science and Physics, in: Lect. Notes Log., vol. 6, Springer-Verlag, 1996, pp. 23-33.

[17] F. Bou, F. Esteva, L. Godo, R. Rodríguez, On the minimum many-valued modal logic over a finite residuated lattice, J. Log. Comput. 21 (5) (2011) 739-790.

[18] B. Chellas, Modal Logic, Cambridge University Press, 1980.

[19] R. Fagin, J. Halpern, Y. Moses, M. Vardi, Reasoning About Knowledge, MIT Press, 1994.

[20] J. Halpern, Y. Moses, A guide to completeness and complexity for modal logics of knowledge and belief, Artif. Intell. 54 (1992) $459-476$.

[21] D. Mundici, Satisfiability in many-valued sentential logic is NP-complete, Theor. Comput. Sci. 52 (5) (1987) 145-153.

[22] F. Bou, M. Cerami, F. Esteva, Finite-valued Łukasiewicz modal logic is pspace-complete, in: Proceedings of the 22th International Joint Conference on Artificial Intelligence, 2011, pp. 774-779.

[23] D. Dubois, P. Hájek, H. Prade, Knowledge-driven versus data-driven logics, J. Log. Lang. Inf. 9 (2000) 65-89.

[24] Mohua Banerjee, Didier Dubois, A simple logic for reasoning about incomplete knowledge, Int. J. Approx. Reason. 55 (2014) $639-653$.

[25] M. Fitting, Many-valued modal logics, in: Fundamenta Informaticae, 1992, pp. 365-448.

[26] M. Fitting, Many-valued modal logics II, Fundamenta Informaticae 17.

[27] G. Hansoul, B. Teheux, Extending Łukasiewicz logics with a modality: algebraic approach to relational semantics, Stud. Log. 101 (3) (2013) $505-545$

[28] P. Hájek, On fuzzy modal logics S5(C), Fuzzy Sets Syst. 161 (18) (2010) 2389-2396.

[29] Y. Maruyama, Reasoning about fuzzy belief and common belief: with emphasis on incomparable beliefs, in: Proceedings of the 22th International Joint Conference on Artificial Intelligence, 2011, pp. 1008-1013.

[30] R. Reiter, A logic for default reasoning, Artif. Intell. 13 (1-2) (1980) 81-132.

[31] M. Fitting, Many-valued non-monotonic modal logics, in: Logic Foundations of Computer Science, 1992, pp. 139-150.

[32] V. Lifschitz, G. Schwarz, Extended logic programs as autoepistemic theories, in: Proceedings of the Second International Workshop on Logic Programming and Nonmonotonic Reasoning, 1993, pp. 101-114.

[33] C. Koutras, S. Zachos, Many-valued reflexive autoepistemic logic, Log. J. IGPL 8 (1) (2000) 403-418. 